\title{
Spectral Galerkin Method in Space and Time for the 2D $g$-Navier-Stokes Equations
}

\author{
Dao Trong Quyet \\ Faculty of Information Technology, Le Quy Don Technical University, 100 Hoang Quoc Viet, Cau Giay, Hanoi 10000, Vietnam \\ Correspondence should be addressed to Dao Trong Quyet; dtq100780@gmail.com
}

Received 20 March 2013; Accepted 20 June 2013

Academic Editor: Chengjian Zhang

Copyright (C) 2013 Dao Trong Quyet. This is an open access article distributed under the Creative Commons Attribution License, which permits unrestricted use, distribution, and reproduction in any medium, provided the original work is properly cited.

We prove the $H^{2}$-stability and $L^{2}$-error analysis of the spectral Galerkin method in space and time with the implicit/explicit Euler scheme for the $2 \mathrm{D} g$-Navier-Stokes equations in bounded domains when the initial data belong to $H^{1}$.

\section{Introduction}

Let $\Omega$ be a bounded domain in $\mathbb{R}^{2}$ with sufficiently smooth boundary $\Gamma$. In this paper, we study the spectral Galerkin method in space and time for the following $2 \mathrm{D} g$-NavierStokes equations:

$$
\begin{gathered}
\frac{\partial u}{\partial t}-v \Delta u+(u \cdot \nabla) u+\nabla p=f(x, t) \quad \text { in }(0,+\infty) \times \Omega, \\
\nabla \cdot(g u)=0 \quad \text { in }(0,+\infty) \times \Omega, \\
u=0 \quad \text { on }(0,+\infty) \times \Gamma, \\
u(0, x)=u_{0}(x), \quad x \in \Omega,
\end{gathered}
$$

where $u=u(x, t)=\left(u_{1}, u_{2}\right)$ is the unknown velocity vector, $p=p(x, t)$ is the unknown pressure, $v>0$ is the kinematic viscosity coefficient, and $u_{0}$ is the initial velocity.

The $g$-Navier-Stokes equations are a variation of the standard Navier-Stokes equations. More precisely, when $g \equiv$ const we get the usual Navier-Stokes equations. The 2D $g$ Navier-Stokes equations arise in a natural way when we study the standard 3D problem in thin domains. We refer the reader to [1] for a derivation of the 2D $g$-Navier-Stokes equations from the 3D Navier-Stokes equations and a relationship between them. As mentioned in [1], good properties of the 2D $g$-Navier-Stokes equations can lead to an initiate of the study of the Navier-Stokes equations on the thin threedimensional domain $\Omega_{g}=\Omega \times(0, g)$. In the last few years, the existence and long-time behavior of both weak and strong solutions to the 2D g-Navier-Stokes equations have been studied extensively (cf. [2-9]). In this paper, we aim to study numerical approximation of the strong solutions to problem (1). To do this, we assume that

(G) $g \in W^{1, \infty}(\Omega)$ such that

$$
\begin{gathered}
0<m_{0} \leq g(x) \leq M_{0} \\
\forall x=\left(x_{1}, x_{2}\right) \in \Omega, \\
|\nabla g|_{\infty}<m_{0} \lambda_{1}^{1 / 2},
\end{gathered}
$$

where $\lambda_{1}>0$ is the first eigenvalue of the $g$ Stokes operator in $\Omega$ (i.e., the operator $A$ defined in Section 2.1 below);

(F) $f \in W^{1, \infty}\left(\mathbb{R}^{+} ; H_{g}\right)$; that is, $f, f_{t} \in L^{\infty}\left(\mathbb{R}^{+} ; H_{g}\right)$.

In this paper, in order to study the numerical approximation of strong solutions to the $2 \mathrm{D} g$-Navier-Stokes equations we will use the spectral Galerkin method in space and time, which is based on the eigen-subspaces of the $g$-Stokes operator. As mentioned in [10] for the Navier-Stokes equations, this method enables us to avoid solving the fully nonlinear $g$-Navier-Stokes equations on the low-frequency subspace, whereas to obtain the low-frequency component of the numerical solution, the usual multilevel spectral methods 
and the postprocessing Galerkin methods need to solve the fully nonlinear $g$-Navier-Stokes equations on the low-frequency subspace. In what follows, we will explain the spectral Galerkin method used in the paper. For the related function spaces, we refer the reader to Section 2.1.

Let $w_{1}, w_{2}, \ldots$ and $\lambda_{1}, \lambda_{2}, \ldots$ be the eigenvectors and eigenvalues of the $g$-Stokes operator. For a fixed integer $m$, let $P_{m}$ be the orthogonal projection of $H_{g}$ onto $H_{m}=$ $\operatorname{span}\left\{w_{1}, \ldots, w_{m}\right\}$. Then, the spectral Galerkin method in space is defined as follows: find $u_{m}(t) \in H_{m}$ such that

$$
\begin{array}{r}
u_{m t}+v A u_{m}+v C u_{m}+P_{m} B\left(u_{m}, u_{m}\right)=P_{m} f \\
t>0, u_{m}(0)=P_{m} u_{0} .
\end{array}
$$

In order to simplify the implementation of the scheme, we restrict ourselves to the semi-implicit Euler scheme applied to the spectral Galerkin method in space. We consider a spectral Galerkin method in space and time with the implicit/explicit Euler scheme: find $u_{m}^{n+1}(n \geq 0)$ such that

$$
\begin{array}{r}
\frac{1}{\Delta t}\left(u_{m}^{n+1}-u_{m}^{n}\right)+\nu A u_{m}^{n+1}+\nu C u_{m}^{n+1}+P_{m} B\left(u_{m}^{n}, u_{m}^{n}\right)=P_{m} f^{n+1}, \\
t>0, u_{m}^{0}=P_{m} u_{0},
\end{array}
$$

where $\Delta t>0$ is the time step size and

$$
f^{n+1}=\frac{1}{\Delta t} \int_{t_{n}}^{t_{n+1}} f(t) d t, \quad t_{n}=n \Delta t
$$

Here, the linear term is treated implicitly to avoid serve time step limitations, whereas the nonlinear term is kept explicitly so that the corresponding discrete system is easily invertible. It is well known that this type of scheme is only stable under some restriction on the time step size. We will obtain $H^{2}$-stability uniform in time stated in Theorem 13 , provided that the following condition holds

$$
C \Delta t \ln \frac{\lambda_{m}}{\lambda_{1}} \leq 1
$$

for some positive constant $C$ depending on the data $\left(u_{0}, v, f\right.$, $\Omega$ ). As mentioned in [11] for the case of $2 \mathrm{D}$ Navier-Stokes equations, the stability condition (6) is a significant improvement compared with the results provided by the nonlinear Galerkin method [12] and the multilevel method [13, 14].

We also derive an $L^{2}$-error estimate of the numerical solution $u_{m}^{n}$ under the stability condition (6):

$$
\left|u\left(t_{n}\right)-u_{m}^{n}\right|^{2} \leq \mathscr{K} \tau^{-1}\left(t_{n}\right) e^{\left(16 / v^{3} \gamma_{0}^{3} \lambda_{1}\right) c_{0}^{2} C_{f}^{2} t_{n}}\left(\lambda_{m+1}^{-2}+\Delta t^{2}\right)
$$

$\forall n \geq 1$, where $C_{f}=\sup _{t \geq 0}|f(t)|, \tau(t)=\min \{1, t\}$ and $\mathscr{K}$ denotes a general positive constant depending only on the data $(\nu, \Omega$, $\left.|\nabla g|_{\infty}, \lambda_{1}, C_{f},\left\|u_{0}\right\|\right)$. Noting that $\tau^{-1}\left(t_{n}\right)$ is a singular factor near $t=0$.

Compared to He's works [11] on the spectral method of the 2D Navier-Stokes equations, here we have to address some additional difficulties. Firstly, to treat the more general condition $\nabla \cdot(g u)=0$, instead of the usual function spaces used for the Navier-Stokes equations, we use the function spaces $H_{g}, V_{g}$ which are defined suitably for the $g$-NavierStokes equations (see Section 2.1 for details). Secondly, we have to deal with the term $\mathrm{Cu}$ in the equation, which only appears for the $g$-Navier-Stokes equations. It is worthy noticing that when $g \equiv 1$, we of course recover the results for the Navier-Stokes equations in [11].

The paper is organized as follows. In the next section, we recall some results on function spaces and inequalities for the nonlinear terms related to the $g$-Navier-Stokes equations, and some discrete Gronwall inequalities are frequently used later. In Section 3, we prove several estimates for the strong solution and the Galerkin approximate solutions of problem (1). In Section 4, we study the error analysis of the spectral Galerkin method in space. Stability and error analysis of the spectral Galerkin method in space and time are discussed in the last section.

\section{Preliminaries}

2.1. Function Spaces and Operators. Let $L^{2}(\Omega, g)=\left(L^{2}(\Omega)\right)^{2}$ and $H_{0}^{1}(\Omega, g)=\left(H_{0}^{1}(\Omega)\right)^{2}$ be endowed, respectively, with the inner products

$$
\begin{array}{r}
(u, v)_{g}=\int_{\Omega} u \cdot v g d x, \quad u, v \in L^{2}(\Omega, g), \\
((u, v))_{g}=\int_{\Omega} \sum_{j=1}^{2} \nabla u_{j} \cdot \nabla v_{j} g d x, \quad u=\left(u_{1}, u_{2}\right), \\
v=\left(v_{1}, v_{2}\right) \in H_{0}^{1}(\Omega, g),
\end{array}
$$

and norms $|u|^{2}=(u, u)_{g},\|u\|^{2}=((u, u))_{g}$. Thanks to assumption (G), the norms $|\cdot|$ and $\|\cdot\|$ are equivalent to the usual ones in $\left(L^{2}(\Omega)\right)^{2}$ and in $\left(H_{0}^{1}(\Omega)\right)^{2}$.

Let

$$
\mathscr{V}=\left\{u \in\left(C_{0}^{\infty}(\Omega)\right)^{2}: \nabla \cdot(g u)=0\right\}
$$

Denote by $H_{g}$ the closure of $\mathscr{V}$ in $L^{2}(\Omega, g)$, and denote by $V_{g}$ the closure of $\mathscr{V}$ in $H_{0}^{1}(\Omega, g)$. It follows that $V_{g} \subset H_{g} \equiv$ $H_{g}^{\prime} \subset V_{g}^{\prime}$, where the injections are dense and continuous. We will use $\|\cdot\|_{*}$ for the norm in $V_{g}^{\prime}$, and $\langle\cdot, \cdot\rangle$ for duality pairing between $V_{g}$ and $V_{g}^{\prime}$. 
We now define the trilinear form $b$ by

$$
b(u, v, w)=\sum_{i, j=1}^{2} \int_{\Omega} u_{i} \frac{\partial v_{j}}{\partial x_{i}} w_{j} g d x
$$

whenever the integrals make sense. It is easy to check that if $u, v, w \in V_{g}$, then

$$
b(u, v, w)=-b(u, w, v)
$$

Hence

$$
b(u, v, v)=0, \quad \forall u, v \in V_{g} .
$$

Set $A: V_{g} \rightarrow V_{g}^{\prime}$ by $\langle A u, v\rangle=((u, v))_{g}, B: V_{g} \times V_{g} \rightarrow$ $V_{g}^{\prime}$ by $\langle B(u, v), w\rangle=b(u, v, w)$. Denote $D(A)=\left\{u \in V_{g}\right.$ : $\left.A u \in H_{g}\right\}$, then $D(A)=H^{2}(\Omega, g) \cap V_{g}$ and $A u=-P_{g} \Delta u$, for all $u \in D(A)$, where $P_{g}$ is the ortho-projector from $L^{2}(\Omega, g)$ onto $H_{g}$. Consequently, there exists an orthogonal basis of $H_{g}$ consisting of the eigenvectors $w_{j}$ of $A$ :

$$
\begin{array}{r}
A w_{j}=\lambda_{j} w_{j}, \quad 0<\lambda_{1} \leq \lambda_{2} \leq \cdots, \\
\lambda_{j} \longrightarrow \infty \text { as } j \longrightarrow \infty .
\end{array}
$$

Furthermore, we can also define the sth power $A^{s}$ of $A$ for all $s \in \mathbb{R}$. The space $D\left(A^{s}\right)$ is the Hilbert space when equipped with the scalar product $\left(A^{s}, A^{s}\right)$ and norm $\left|A^{s \cdot}\right|$, where $(\cdot, \cdot)$ and $|\cdot|$ denote the scalar product and norm in $H_{g}$. In particular, $D\left(A^{0}\right)=H_{g}$ and $D\left(A^{1 / 2}\right)=V_{g}$.

Let $H_{m}=\operatorname{span}\left\{w_{1}, \ldots, w_{m}\right\}$. Then, the following estimates hold:

$$
\begin{array}{r}
\lambda_{1}|v|^{2} \leq\|v\|^{2} \quad \forall v \in V_{g}, \quad\left\|v_{m}\right\|^{2} \leq \lambda_{m}\left|v_{m}\right|^{2} \\
\forall v_{m} \in H_{m}, \\
\lambda_{m+1}\left|w_{m}\right|^{2} \leq\left\|w_{m}\right\|^{2} \quad \forall w_{m} \in V_{g} \backslash H_{m} .
\end{array}
$$

Using the Hölder inequality, the Ladyzhenskaya inequality (when $n=2)$ :

$$
|u|_{L^{4}} \leq c|u|^{1 / 2}|\nabla u|^{1 / 2}, \quad \forall u \in H_{0}^{1}(\Omega)
$$

and the interpolation inequalities, as in $[15,16]$, one can prove the following.
Lemma 1. If $n=2$, then

$$
\begin{aligned}
& |b(u, v, w)| \\
& \leq\left\{\begin{array}{r}
c_{0}|u|^{1 / 2}\|u\|^{1 / 2}\|v\||w|^{1 / 2}\|w\|^{1 / 2}, \\
\forall u, v, w \in V_{g}, \\
c_{0}|u|^{1 / 2}\|u\|^{1 / 2}\|v\||A w|^{1 / 2}|w|^{1 / 2}, \\
\forall u \in V_{g}, v \in D(A), w \in H_{g}, \\
c_{0}|u|^{1 / 2}|A u|^{1 / 2}\|v\||w|, \\
\forall u \in D(A), \quad v \in V_{g}, w \in H_{g}, \\
c_{0}|u|\|v\||w|^{1 / 2}|A w|^{1 / 2}, \\
\forall u \in H_{g} v \in V_{g}, w \in D(A),
\end{array}\right.
\end{aligned}
$$

$$
\begin{array}{r}
|b(u, v, w)|+|b(w, v, u)| \leq c_{0}|u|^{1 / 2}\|u\|^{1 / 2}\|v\|^{1 / 2}|A v|^{1 / 2}|w| \\
\forall u \in V_{g}, \quad v \in D(A), w \in H_{g}
\end{array}
$$

$$
\begin{aligned}
& |b(u, v, w)|+|b(w, v, u)| \\
& \leq c_{0}\left(1+\ln \frac{|A u|^{2}}{\lambda_{1}\|u\|^{2}}\right)^{1 / 2}\|u\|\|v\||w| \\
& \forall u \in D(A), \quad v \in V_{g}, w \in H_{g},
\end{aligned}
$$

where $c_{0}$ are appropriate constants depending only on $\Omega$.

Lemma 2 (see [3]). Let $u \in L^{2}(0, T ; D(A)) \cap L^{\infty}\left(0, T ; V_{g}\right)$, then the function Bu defined by

$$
(B u(t), v)_{g}=b(u(t), u(t), v), \quad \forall v \in H_{g} \text {, a.e. } t \in[0, T],
$$

belongs to $L^{4}\left(0, T ; H_{g}\right)$, and therefore also belongs to $L^{2}(0, T$; $\left.H_{g}\right)$.

Lemma 3 (see [4]). Let $u \in L^{2}\left(0, T ; V_{g}\right)$, then the function $C u$ defined by

$$
\begin{aligned}
(C u(t), v)_{g} & =\left(\left(\frac{\nabla g}{g} \cdot \nabla\right) u, v\right)_{g} \\
& =b\left(\frac{\nabla g}{g}, u, v\right), \quad \forall v \in V_{g},
\end{aligned}
$$

belongs to $L^{2}\left(0, T ; H_{g}\right)$, and hence also belongs to $L^{2}\left(0, T ; V_{g}^{\prime}\right)$. Moreover,

$|C u(t)| \leq \frac{|\nabla g|_{\infty}}{m_{0}} \cdot\|u(t)\|, \quad$ for a.e. $t \in(0, T)$,

$\|C u(t)\|_{*} \leq \frac{|\nabla g|_{\infty}}{m_{0} \lambda_{1}^{1 / 2}} \cdot\|u(t)\|, \quad$ for a.e. $t \in(0, T)$.

Since

$$
-\frac{1}{g}(\nabla \cdot g \nabla) u=-\Delta u-\left(\frac{\nabla g}{g} \cdot \nabla\right) u,
$$


we have

$$
\begin{aligned}
(-\Delta u, v)_{g} & =((u, v))_{g}+\left(\left(\frac{\nabla g}{g} \cdot \nabla\right) u, v\right)_{g} \\
& =(A u, v)_{g}+\left(\left(\frac{\nabla g}{g} \cdot \nabla\right) u, v\right)_{g}, \quad \forall u, v \in V_{g} .
\end{aligned}
$$

2.2. Discrete Gronwall Inequalities. Hereafter, we will frequently use the following modified discrete Gronwall lemmas.

Lemma 4 (see [12]). Let $0<\Delta t<1, a_{k}, g_{k}, h_{k}$ for integers $k \geq 0$ be nonnegative numbers such that

$$
\frac{1}{\Delta t}\left(a_{k+1}-a_{k}\right) \leq g_{k} a_{k}+h_{k} \quad \forall 0 \leq k \leq J .
$$

If

$$
\Delta t \sum_{k=0}^{J} g_{k} \leq \alpha_{1}, \quad \Delta t \sum_{k=0}^{J} h_{k} \leq \alpha_{2},
$$

for $J \leq n_{0}-1$ and

$$
\begin{gathered}
\Delta t \sum_{k=k_{0}}^{k_{0}+n_{0}-1} g_{k} \leq \alpha_{1}, \quad \Delta t \sum_{k=k_{0}}^{k_{0}+n_{0}-1} h_{k} \leq \alpha_{2}, \\
\Delta t \sum_{k=k_{0}}^{k_{0}+n_{0}-1} a_{k} \leq a_{0}+\alpha_{3},
\end{gathered}
$$

for $J \geq n_{0}, 0 \leq k_{0} \leq J+1-n_{0}$, then

$$
a_{J+1} \leq e^{\alpha_{1}}\left(a_{0}+\alpha_{2}+\alpha_{3}\right) .
$$

Lemma 5 (see [13]). Let $\alpha$ and $a_{k}, b_{k}, h_{k}, k \geq 0$ be nonnegative numbers such that

$$
(1+\gamma \Delta t) a_{k+1}-a_{k}+b_{k+1} \Delta t \leq h_{k} \Delta t \quad \forall 0 \leq k \leq J .
$$

Then,

$$
\begin{aligned}
a_{J+1} & +\Delta t \sum_{k=1}^{J+1}(1+\gamma \Delta t)^{-(J+2-k)} b_{k} \\
& \leq(1+\gamma \Delta t)^{-(J+1)} a_{0}+\Delta t \sum_{k=0}^{J}(1+\gamma \Delta t)^{-(J+1-k)} h_{k} .
\end{aligned}
$$

Lemma 6 (see [11]). Let $\beta$ and $a_{k}, b_{k}, g_{k}, h_{k}$ for integers $k \geq 0$ be nonnegative numbers such that

$$
a_{n}+\Delta t \sum_{k=0}^{n} b_{k} \leq \Delta t \sum_{k=0}^{n} g_{k} a_{k}+\Delta t \sum_{k=0}^{n} h_{k}+\beta \quad \forall n \geq 0 .
$$

Suppose that $g_{k} \Delta t<1$, for all $k \geq 0$, and set $\sigma_{k}=\left(1-g_{k} \Delta t\right)^{-1}$. Then,

$$
a_{n}+\Delta t \sum_{k=0}^{n} b_{k} \leq \exp \left\{\Delta t \sum_{k=0}^{n} \sigma_{k} g_{k}\right\}\left(\Delta t \sum_{k=0}^{n} h_{k}+\beta\right) \quad \forall n \geq 0 .
$$

Moreover, if

$$
a_{r}+\Delta t \sum_{k=r}^{n} b_{k} \leq \Delta t \sum_{k=r}^{n} g_{k} a_{k}+\Delta t \sum_{k=r}^{n} h_{k}+\beta \quad \forall 0 \leq r \leq n,
$$

and $g_{k} \Delta t<1$ for all $0 \leq k \leq n$, then

$$
\begin{array}{r}
a_{r}+\Delta t \sum_{k=r}^{n} b_{k} \leq \exp \left\{\Delta t \sum_{k=0}^{n} \sigma_{k} g_{k}\right\}\left(\Delta t \sum_{k=0}^{n} h_{k}+\beta\right) ; \\
\forall 0 \leq r \leq n .
\end{array}
$$

\section{Existence and Some Estimates of Strong Solutions}

In this section, we will prove some estimates for the strong solution $u$ and the Galerkin approximate solutions $u_{m}$ of problem (1). First, with the operators defined in Section 2.1, one can write this problem as follows:

$$
\frac{d u}{d t}+v A u+v C u+B(u, u)=f(t), \quad t>0, u(0)=u_{0} .
$$

Definition 7. For $u_{0} \in V_{g}$ given, a strong solution of problem (1) is a function $u \in L^{2}(0, T ; D(A)) \cap C\left([0, T] ; V_{q}\right)$ for all $T>0$ such that $u(0)=u_{0}$, and $u$ satisfies (35) in $H_{g}$ for a.e. $t>0$.

Theorem 8. Suppose that $f, f_{t} \in L^{\infty}\left(\mathbb{R}^{+} ; H_{g}\right)$ and $u_{0} \in V_{g}$. Then, problem (1) has a unique strong solution $u$ satisfying the following estimates for all $t>0$,

$$
\begin{gathered}
|u(t)|^{2} \leq e^{-v \gamma_{0} \lambda_{1} t}\left|u_{0}\right|^{2}+\frac{1}{v^{2} \gamma_{0}^{2} \lambda_{1}^{2}} C_{f}^{2}, \\
\int_{0}^{t} e^{\nu \gamma_{0} \lambda_{1} s}\|u\|^{2} d s \leq \frac{2}{v \gamma_{0}}\left|u_{0}\right|^{2}+\frac{4}{\nu^{3} \gamma_{0}^{3} \lambda_{1}^{2}} e^{\nu \gamma_{0} \lambda_{1} t} C_{f}^{2}, \\
\int_{0}^{t}\|u(s)\|^{2} d s \leq \frac{1}{\nu \gamma_{0}}\left|u_{0}\right|^{2}+\frac{t C_{f}^{2}}{\nu^{2} \gamma_{0}^{2} \lambda_{1}}, \\
\|u(t)\|^{2} \leq C_{u_{0}, f}, \\
\tau(t)\left(\left|u_{t}(t)\right|^{2}+|A u(t)|^{2}\right)+\tau^{2}(t)\left\|u_{t}(t)\right\|^{2} \leq \mathscr{K}, \\
\int_{0}^{t} e^{-\gamma \gamma_{0} \lambda_{1}(t-s)}\left(|A u|^{2}+\left|u_{t}\right|^{2}+\tau(s)\left\|u_{t}\right\|^{2}\right. \\
\left.+\tau^{2}(s)\left|u_{t t}\right|^{2}+\tau^{2}(s)\left|A u_{t}\right|^{2}\right) d s \leq \mathscr{K},
\end{gathered}
$$

where $\gamma_{0}=1-\left(|\nabla g|_{\infty} / m_{0} \lambda_{1}^{1 / 2}\right)>0, \tau(t)=\min \{1, t\}, C_{f}=$ $\sup _{t \geq 0}|f|, C_{u_{0}, f}=C\left(\left\|u_{0}\right\|, C_{f}\right)$, and $\mathscr{K}$ is a generic positive constant depending only on the data $\left(\nu, \Omega,|\nabla g|_{\infty}, \lambda_{1}, C_{f}\right.$, $\left.\left\|u_{0}\right\|\right)$. Moreover, all above estimates are also valid for the Galerkin approximate solutions $u_{m}$ of problem (35).

Proof. We refer to [3] for the proof of existence and uniqueness of the strong solution $u$ and estimates (36)-(38). We now prove (39)-(40). 
First, we take the scalar product of (35) with $e^{\nu \gamma_{0} \lambda_{1} t} A u$ and $v^{-1} e^{\nu \gamma_{0} \lambda_{1} t} u_{t}$, respectively, and add the resulting relations to obtain

$$
\begin{aligned}
& \frac{d}{d t}\left(e^{\nu \gamma_{0} \lambda_{1} t}\|u\|^{2}\right)+e^{\nu \gamma_{0} \lambda_{1} t}\left(\nu|A u|^{2}+\nu^{-1}\left|u_{t}\right|^{2}\right) \\
& \quad+e^{\nu \gamma_{0} \lambda_{1} t} b\left(u, u, A u+\nu^{-1} u_{t}\right) \\
& \quad+\nu e^{\nu \gamma_{0} \lambda_{1} t}\left(C u, A u+\nu^{-1} u_{t}\right)_{g} \\
& =\nu \gamma_{0} \lambda_{1} e^{\nu \lambda_{1} t}\|u\|^{2}+e^{\nu \gamma_{0} \lambda_{1} t}\left(f, A u+\nu^{-1} u_{t}\right) .
\end{aligned}
$$

Using Lemmas 1 and 3 and Cauchy's inequality, we have

$$
\begin{gathered}
\left|b\left(u, u, A u+v^{-1} u_{t}\right)\right| \leq \frac{1}{4}\left(\nu|A u|^{2}+v^{-1}\left|u_{t}\right|^{2}\right)+c|u|^{2}\|u\|^{2}, \\
\left|v\left(C u, A u+v^{-1} u_{t}\right)_{g}\right| \leq \frac{1}{8}\left(\nu|A u|^{2}+v^{-1}\left|u_{t}\right|^{2}\right)+c\|u\|^{2}, \\
\left|\left(f, A u+v^{-1} u_{t}\right)\right| \leq \frac{1}{8}\left(\nu|A u|^{2}+v^{-1}\left|u_{t}\right|^{2}\right)+c|f|^{2} .
\end{gathered}
$$

By combining these inequalities with (41), we get

$$
\begin{aligned}
& \frac{d}{d t}\left(e^{\nu \gamma_{0} \lambda_{1} t}\|u\|^{2}\right)+\frac{1}{2} e^{\nu \gamma_{0} \lambda_{1} t}\left(\nu|A u|^{2}+\nu^{-1}\left|u_{t}\right|^{2}\right) \\
& \quad \leq e^{\nu \gamma_{0} \lambda_{1} t}\left(\nu \lambda_{1}+c|u|^{2}\|u\|^{2}+c\right)\|u\|^{2}+c e^{\nu \gamma_{0} \lambda_{1} t}|f|^{2} .
\end{aligned}
$$

Integrating (43) from 0 to $t$ and using (36)-(38), we obtain, after multiplying by $e^{-\gamma \gamma_{0} \lambda_{1} t}$, that

$$
e^{-\gamma \gamma_{0} \lambda_{1} t} \int_{0}^{t} e^{\nu \gamma_{0} \lambda_{1} s} \tau(s)\left(\nu|A u|^{2}+\nu^{-1}\left|u_{t}\right|^{2}\right) d s \leq \mathscr{K} \quad \forall t \geq 0 .
$$

In view of (44), there exists a sequence $\epsilon_{k} \rightarrow 0$ such that

$$
\tau^{2}\left(\epsilon_{k}\right)\left(\nu\left|A u\left(\epsilon_{k}\right)\right|^{2}+\nu^{-1}\left|u_{t}\left(\epsilon_{k}\right)\right|^{2}\right) \longrightarrow 0 .
$$

Now, differentiating (35) with respect to $t$ yields

$$
u_{t t}+v A u_{t}+\nu C u_{t}+B\left(u_{t}, u\right)+B\left(u, u_{t}\right)=f_{t} .
$$

We take the scalar product (46) with $2 u_{t}$ to obtain

$$
\begin{aligned}
& \frac{d}{d t}\left|u_{t}\right|^{2}+2 v\left\|u_{t}\right\|^{2}+2 v\left(C u_{t}, u_{t}\right)_{g} \\
& +2 b\left(u_{t}, u, u_{t}\right)+2 b\left(u, u_{t}, u_{t}\right)=2\left(f_{t}, u_{t}\right)_{g} .
\end{aligned}
$$

By Lemma 3, we have

$$
\begin{aligned}
& \frac{d}{d t}\left|u_{t}\right|^{2}+2 v\left\|u_{t}\right\|^{2} \\
& \leq 2 v \frac{|\nabla g|_{\infty}}{m_{0} \lambda_{1}^{1 / 2}}\left\|u_{t}\right\|^{2}+2\left|b\left(u_{t}, u, u_{t}\right)\right| \\
& \quad+2\left|b\left(u, u_{t}, u_{t}\right)\right|+2\left(f_{t}, u_{t}\right)_{g} .
\end{aligned}
$$

Using Lemma 1 and Cauchy's inequality, we get

$$
\frac{d}{d t}\left|u_{t}\right|^{2}+v \gamma_{0}\left\|u_{t}\right\|^{2} \leq c\left|u_{t}\right|^{2}\|u\|^{2}+c\left|f_{t}\right|^{2} .
$$

Multiplying the last inequality by $\tau(t) e^{\nu \gamma_{0} \lambda_{1} t}$, we have

$$
\begin{aligned}
& \frac{d}{d t}\left(e^{\nu \gamma_{0} \lambda_{1} t} \tau(t)\left|u_{t}\right|^{2}\right) \\
& \quad+\nu \gamma_{0} e^{\nu \gamma_{0} \lambda_{1} t} \tau(t)\left\|u_{t}\right\|^{2} \\
& \leq e^{\nu \gamma_{0} \lambda_{1} t}\left(1+\nu \gamma_{0} \lambda_{1}+c\|u\|^{2}\right)\left|u_{t}\right|^{2}+c e^{\nu \gamma_{0} \lambda_{1} t}\left|f_{t}\right|^{2} .
\end{aligned}
$$

Therefore, integrating (50) from $\epsilon_{k}$ to $t$, letting $\epsilon_{k} \rightarrow 0$, and using (44) and (45), one finds, after multiplying by $e^{-\gamma \gamma_{0} \lambda_{1} t}$, that

$$
\tau(t)\left|u_{t}(t)\right|^{2}+v e^{-\nu \gamma_{0} \lambda_{1} t} \int_{0}^{t} e^{\nu \gamma_{0} \lambda_{1} s} \tau(s)\left\|u_{t}\right\|^{2} d s \leq \mathscr{K} \quad \forall t \geq 0 .
$$

Moreover, in view of (35), (36)-(38), and (51), we see that

$$
\begin{aligned}
\tau(t)|A u(t)|^{2} \leq & c\left|f_{t}(t)\right|^{2}+c \tau(t)\left|u_{t}(t)\right|^{2} \\
& +c|u(t)|^{2}\|u(t)\|^{4} \leq \mathscr{K} \quad \forall t \geq 0 .
\end{aligned}
$$

Also, in view of (51), there exists a sequence $\epsilon_{k} \rightarrow 0$ such that

$$
\tau^{2}\left(\epsilon_{k}\right)\left\|u_{t}\left(\epsilon_{k}\right)\right\|^{2} \longrightarrow 0 .
$$

We again take the scalar product (46) with $2 A u_{t}$ to obtain

$$
\begin{aligned}
\frac{d}{d t}\left\|u_{t}\right\|^{2}+2 v\left|A u_{t}\right|^{2}+2 v\left(C u_{t}, A u_{t}\right)_{g} \\
+2 b\left(u_{t}, u, A u_{t}\right)+2 b\left(u, u_{t}, A u_{t}\right)=2\left(f_{t}, A u_{t}\right)_{g} .
\end{aligned}
$$

By Lemma 3 and Cauchy's inequality, we have

$$
\begin{aligned}
\frac{d}{d t}\left\|u_{t}\right\|^{2} & +2 v\left|A u_{t}\right|^{2} \\
\leq & 2 \nu \frac{|\nabla g|_{\infty}}{m_{0} \lambda_{1}^{1 / 2}}\left|A u_{t}\right|^{2}+\nu \frac{|\nabla g|_{\infty} \lambda_{1}^{1 / 2}}{2 m_{0}}\left\|u_{t}\right\|^{2} \\
& +2 b\left|\left(u_{t}, u, A u_{t}\right)\right|+2\left|b\left(u, u_{t}, A u_{t}\right)\right|+2\left(f_{t}, A u_{t}\right)_{g} .
\end{aligned}
$$

Using Lemma 1, Cauchy's inequality, and Young's inequality, we obtain

$$
\begin{aligned}
& \frac{d}{d t}\left\|u_{t}\right\|^{2}+v \gamma_{0}\left|A u_{t}\right|^{2} \\
& \quad \leq c\|u\|^{4}\left\|u_{t}\right\|^{2}+c\left|f_{t}\right|^{2}+v \frac{|\nabla g|_{\infty} \lambda_{1}^{1 / 2}}{2 m_{0}}\left\|u_{t}\right\|^{2} .
\end{aligned}
$$


Multiplying the last inequality by $\tau^{2}(t) e^{\nu \gamma_{0} \lambda_{1} t}$, we have

$$
\begin{aligned}
& \frac{d}{d t}\left(e^{\nu \gamma_{0} \lambda_{1} t} \tau^{2}(t)\left\|u_{t}\right\|^{2}\right)+\nu \gamma_{0} \tau^{2}(t) e^{\nu \gamma_{0} \lambda_{1} t}\left|A u_{t}\right|^{2} \\
& \leq c e^{\nu \gamma_{0} \lambda_{1} t}\left|f_{t}\right|^{2} \\
& \quad+e^{\nu \gamma_{0} \lambda_{1} t}\left(2+\nu \gamma_{0} \lambda_{1}+c\|u\|^{4}+\frac{\nu|\nabla g|_{\infty} \lambda_{1}^{1 / 2}}{2 m_{0}}\right) \tau(t)\left\|u_{t}\right\|^{2} .
\end{aligned}
$$

Integrating (57) from $\epsilon_{k}$ to $t$, letting $\epsilon_{k} \rightarrow 0$, and using (36)(38), (51), and (53), we obtain, after a final multiplication by $e^{-v \gamma_{0} \lambda_{1} t}$

$$
\begin{array}{r}
\tau^{2}(t)\left\|u_{t}(t)\right\|^{2}+\nu \gamma_{0} e^{-v \gamma_{0} \lambda_{1} t} \int_{0}^{t} e^{\nu \gamma_{0} \lambda_{1} s} \tau^{2}(s)\left|A u_{t}\right|^{2} d s \leq \mathscr{K} \\
\forall t \geq 0 .
\end{array}
$$

Using Lemmas 1 and 3 and (46), we deduce that

$$
\begin{aligned}
& e^{-\nu \gamma_{0} \lambda_{1} t} \int_{0}^{t} \tau^{2}(t) e^{\nu \gamma_{0} \lambda_{1} s}\left|u_{t t}\right|^{2} d s \\
& \leq c e^{-v \gamma_{0} \lambda_{1} t} \\
& \quad \times \int_{0}^{t} \tau^{2}(t) e^{\nu \gamma_{0} \lambda_{1} s}\left(\left|A u_{t}\right|^{2}+\|u\|^{2}\left|A u_{t}\right|^{2}+\left|f_{t}\right|^{2}\right) d s
\end{aligned}
$$

$\forall t \geq 0$.

Combining (44), (51), (52), (58), and (59) yields (39) and (40).

Finally, we observe that the problem for the approximate solution $u_{m}$ is similar to problem (35), and

$$
\left|u_{m}(0)\right| \leq\left|u_{0}\right|, \quad\left\|u_{m}(0)\right\| \leq\left\|u_{0}\right\|,
$$

so $u_{m}$ satisfies the same estimates as those for the strong solution $u$ of problem (35).

\section{Spectral Galerkin Method in Space}

For a fixed integer $m$, let $P_{m}$ be the orthogonal projection of $H_{g}$ onto $H_{m}=\operatorname{span}\left\{w_{1}, \ldots, w_{m}\right\}$ and $Q_{m}=I-P_{m}$. Then, every solution $u$ of problem (1) can be decomposed uniquely into

$$
u=p+q, \quad \text { where } p=P_{m} u, q=Q_{m} u
$$

Now, we apply $P_{m}$ and $Q_{m}$ to (35) to obtain

$$
\begin{array}{ll}
p_{t}+v A p+\nu C p+P_{m} B(u, u)=P_{m} f & \forall t>0, \\
q_{t}+v A q+v C q+Q_{m} B(u, u)=Q_{m} f & \forall t>0
\end{array}
$$

and the initial conditions $p(0)=P_{m} u_{0}, q(0)=Q_{m} u_{0}$.
Using Theorem 8 and the property of $P_{m}$, we arrive at the following estimates of $q(t)=Q_{m} u(t)$ :

$$
\begin{gathered}
|q(t)|^{2} \leq \mathscr{K} \lambda_{m+1}^{-1}, \\
\tau(t)\left(|q(t)|^{2}+\lambda_{m+1}^{-1}\|q(t)\|^{2}\right) \leq 2 \mathscr{K} \lambda_{m+1}^{-2} \quad \forall t \geq 0 \\
e^{-v \gamma_{0} \lambda_{1} t} \int_{0}^{t} e^{v \gamma_{0} \lambda_{1} s}\left(|q|^{2}+\lambda_{m+1}^{-1}\|q\|^{2}\right) d s \leq 2 \mathscr{K} \lambda_{m+1}^{-2} \quad \forall t \geq 0 .
\end{gathered}
$$

We now define the spectral Galerkin method as follows: find $u_{m}(t) \in H_{m}$ such that

$$
u_{m t}+v A u_{m}+v C u_{m}+P_{m} B\left(u_{m}, u_{m}\right)=P_{m} f \quad \forall t>0,
$$

with the initial condition $u_{m}(0)=P_{m} u_{0}$.

In order to give an analysis of the error $u-u_{m}$ in the $L^{2}$-norm, we begin with a technical result concerning a dual linearized $g$-Navier-Stokes problem which is a similar problem to that used in [17]. We consider, for any given $t>0$ and $\xi \in L^{2}\left(0, t ; H_{g}\right)$, the dual problem: find $\Phi(s) \in H_{m}$ such that

$$
\begin{gathered}
\left(v, \Phi_{s}\right)_{g}-a(v, \Phi)-v(C v, \Phi)_{g}-b\left(v, u_{m}, \Phi\right)-b\left(u_{m}, v, \Phi\right) \\
=\left(v, e^{\nu \gamma_{0} \lambda_{1} s} \xi\right)_{g}, \quad 0 \leq s<t,
\end{gathered}
$$

for all $v \in H_{m}$ with $\Phi(t)=0$. It is easy to see that (67) is a wellposed problem and has a unique solution $\Phi \in L^{\infty}\left(0, t ; V_{g}\right) \cap$ $L^{2}(0, t ; D(A))$.

Next, we prove a regularity result of problem (67).

Lemma 9. If $u_{0} \in V_{g}$, then the solution $\Phi(s)$ of problem (67) satisfies

$$
\begin{aligned}
& e^{-\gamma \gamma_{0} \lambda_{1} s}\|\Phi(s)\|^{2}+\int_{s}^{t} e^{-\gamma \gamma_{0} \lambda_{1} r}\left(|A \Phi|^{2}+\left|\Phi_{s}\right|^{2}\right) d r \\
& \leq \mathscr{K} \exp \left\{\frac{2}{\nu \gamma_{0}} c_{0}^{2} \int_{0}^{t}\left\|u_{m}\right\|^{2} d r\right\} \int_{0}^{t} e^{\nu \gamma_{0} \lambda_{1} r}|\xi|^{2} d r .
\end{aligned}
$$

Proof. Taking $v=-2 \Phi$ in (67), we obtain

$$
\begin{gathered}
-\frac{d}{d s}|\Phi|^{2}+2 \nu\|\Phi\|^{2}+2 \nu(C \Phi, \Phi)_{g}+2 b\left(\Phi, u_{m}, \Phi\right) \\
=-2\left(\Phi, e^{\nu \gamma_{0} \lambda_{1} s} \xi\right)_{g} \quad \forall 0 \leq s<t .
\end{gathered}
$$

Using Lemma 3, we have

$$
\begin{array}{r}
-\frac{d}{d s}|\Phi|^{2}+2 \nu \gamma_{0}\|\Phi\|^{2} \leq 2\left|\left(\Phi, e^{\nu \gamma_{0} \lambda_{1} s} \xi\right)_{g}\right|+2\left|b\left(\Phi, u_{m}, \Phi\right)\right| \\
\forall 0 \leq s<t .
\end{array}
$$

Using Lemma 1 and Cauchy's inequality, we get

$$
\begin{gathered}
2\left|b\left(\Phi, u_{m}, \Phi\right)\right| \leq \frac{\nu \gamma_{0}}{2}\|\Phi\|^{2}+\frac{2}{v \gamma_{0}} c_{0}^{2}\left\|u_{m}\right\|^{2}|\Phi|^{2}, \\
2\left|\left(\Phi, e^{\nu \gamma_{0} \lambda_{1} s} \xi\right)_{g}\right| \leq \frac{\nu \gamma_{0}}{4}\|\Phi\|^{2}+\frac{4}{\nu \gamma_{0} \lambda_{1}} e^{2 \nu \gamma_{0} \lambda_{1} s}|\xi|^{2} .
\end{gathered}
$$


Then, we have

$$
\begin{aligned}
& -\frac{d}{d s}|\Phi|^{2}+\frac{5}{4} \nu \gamma_{0}\|\Phi\|^{2} \\
& \quad \leq \frac{2}{\nu \gamma_{0}} c_{0}^{2}\left\|u_{m}\right\|^{2}|\Phi|^{2}+\frac{4}{\nu \gamma_{0} \lambda_{1}} e^{2 v \gamma_{0} \lambda_{1} s}|\xi|^{2} \quad \forall 0 \leq s<t .
\end{aligned}
$$

Multiplying this inequality by $e^{-\gamma \gamma_{0} \lambda_{1} s}$ and using (14), we obtain

$$
\begin{aligned}
& -\frac{d}{d s}\left(e^{-\gamma \gamma_{0} \lambda_{1} s}|\Phi|^{2}\right)+\frac{\nu \gamma_{0}}{4} e^{-\nu \gamma_{0} \lambda_{1} s}\|\Phi\|^{2} \\
& \quad \leq \frac{2}{\nu \gamma_{0}} c_{0}^{2} e^{-\nu \gamma_{0} \lambda_{1} s}\left\|u_{m}\right\|^{2}|\Phi|^{2}+\frac{4}{\nu \gamma_{0} \lambda_{1}} e^{\nu \gamma_{0} \lambda_{1} s}|\xi|^{2} .
\end{aligned}
$$

Multiplying this inequality by $e^{\left(2 / v \gamma_{0}\right) c_{0}^{2} \int_{0}^{s}\left\|u_{m}\right\|^{2} d r}$ yields

$$
\begin{gathered}
-\frac{d}{d s}\left(e^{\left(2 / \nu \gamma_{0}\right) c_{0}^{2} \int_{0}^{s}\left\|u_{m}\right\|^{2} d r} e^{-v \gamma_{0} \lambda_{1} s}|\Phi|^{2}\right) \\
+\frac{\nu \gamma_{0}}{4} e^{\left(2 / \nu \gamma_{0}\right) c_{0}^{2} \int_{0}^{s}\left\|u_{m}\right\|^{2} d r} e^{-\gamma \gamma_{0} \lambda_{1} s}\|\Phi\|^{2} \\
\leq \frac{4}{\nu \gamma_{0} \lambda_{1}} e^{\left(2 / v \gamma_{0}\right) c_{0}^{2} \int_{0}^{s}\left\|u_{m}\right\|^{2} d r} e^{\nu \gamma_{0} \lambda_{1} s}|\xi|^{2} .
\end{gathered}
$$

Integrating (74) from $s$ to $t$ and noting that $\Phi(t)=0$, we obtain, after multiplying by $e^{-\left(2 / v \gamma_{0}\right) c_{0}^{2} \int_{0}^{s}\left\|u_{m}\right\|^{2} d r}$, that

$$
\begin{aligned}
& e^{-v \gamma_{0} \lambda_{1} s}|\Phi(s)|^{2}+\frac{\nu \gamma_{0}}{4} \int_{s}^{t} e^{-v \gamma_{0} \lambda_{1} r}\|\Phi\|^{2} d r \\
& \quad \leq \frac{4}{v \gamma_{0} \lambda_{1}} e^{\left(2 / v \gamma_{0}\right) c_{0}^{2} \int_{0}^{t}\left\|u_{m}\right\|^{2} d r} \int_{0}^{t} e^{\nu \gamma_{0} \lambda_{1} r}|\xi|^{2} d r \quad \forall 0 \leq s \leq t .
\end{aligned}
$$

Moreover, inserting $v=-2 A \Phi$ into (67), we get

$$
\begin{aligned}
-\frac{d}{d t} \| & \|^{2}+2 \nu|A \Phi|^{2}+2 \nu(C A \Phi, \Phi)_{g} \\
& -2\left\{b\left(A \Phi, \Phi, u_{m}\right)+b\left(u_{m}, \Phi, A \Phi\right)\right\} \\
= & -2\left(A \Phi, e^{\nu \gamma_{0} \lambda_{1} s} \xi^{k}\right)_{g} .
\end{aligned}
$$

Using Lemma 3, (14), and Cauchy's inequality, we have

$$
2 v(C A \Phi, \Phi)_{g} \leq 2 v \frac{|\nabla g|_{\infty}}{m_{0} \lambda_{1}^{1 / 2}}|A \Phi|^{2}+\frac{\nu|\nabla g|_{\infty} \lambda_{m}}{2 m_{0} \lambda_{1}^{1 / 2}}\|\Phi\|^{2} .
$$

Therefore,

$$
\begin{aligned}
-\frac{d}{d t}\|\Phi\|^{2}+2 \nu \gamma_{0}|A \Phi|^{2} & \\
\leq & 2\left|\left(A \Phi, e^{\nu \gamma_{0} \lambda_{1} s} \xi^{k}\right)_{g}\right|+2\left|b\left(A \Phi, \Phi, u_{m}\right)+b\left(u_{m}, \Phi, A \Phi\right)\right| \\
& +\frac{\nu|\nabla g|_{\infty} \lambda_{m}}{2 m_{0} \lambda_{1}^{1 / 2}}\|\Phi\|^{2} .
\end{aligned}
$$

Multiplying this inequality, by $e^{-v \gamma_{0} \lambda_{1} s}$ and using (14), we obtain

$$
\begin{aligned}
& -\frac{d}{d t}\left(e^{-\gamma \gamma_{0} \lambda_{1} s}\|\Phi\|^{2}\right)+\nu \gamma_{0} e^{-\gamma \gamma_{0} \lambda_{1} s}|A \Phi|^{2} \\
& \leq 2\left|\left(A \Phi, \xi^{k}\right)_{g}\right| \\
& \quad+2 e^{-\gamma \gamma_{0} \lambda_{1} s}\left|b\left(A \Phi, \Phi, u_{m}\right)+b\left(u_{m}, \Phi, A \Phi\right)\right| \\
& \quad+\frac{\nu|\nabla g|_{\infty} \lambda_{m}}{2 m_{0} \lambda_{1}^{1 / 2}} e^{-\gamma \gamma_{0} \lambda_{1} s}\|\Phi\|^{2} .
\end{aligned}
$$

Using Lemma 1, Cauchy's inequality and Young's inequality, we have

$$
\begin{gathered}
2\left|b\left(A \Phi, \Phi, u_{m}\right)\right|+2\left|b\left(u_{m}, \Phi, A \Phi\right)\right| \\
\leq c_{0}|A \Phi|^{3 / 2}\|\Phi\|^{1 / 2}\left\|u_{m}\right\|^{1 / 2}\left|u_{m}\right|^{1 / 2} \\
\leq \frac{\nu \gamma_{0}}{2}|A \Phi|^{2}+c\|\Phi\|^{2}\left\|u_{m}\right\|^{2}\left|u_{m}\right|^{2}, \\
2\left|\left(A \Phi, \xi^{k}\right)_{g}\right| \leq \frac{v \gamma_{0}}{4} e^{-\nu \gamma_{0} \lambda_{1} s}|A \Phi|^{2}+\frac{4}{\nu \gamma_{0}} e^{\nu \gamma_{0} \lambda_{1} s}\left|\xi^{k}\right|^{2} .
\end{gathered}
$$

Combining the above estimates with (79) yields

$$
\begin{aligned}
& -\frac{d}{d t}\left(e^{-\gamma \gamma_{0} \lambda_{1} s}\|\Phi\|^{2}\right)+\frac{\nu \gamma_{0}}{4} e^{-\nu \gamma_{0} \lambda_{1} s}|A \Phi|^{2} \\
& \leq c e^{-\gamma \gamma_{0} \lambda_{1} s}\|\Phi\|^{2}\left\|u_{m}\right\|^{2}\left|u_{m}\right|^{2} \\
& \quad+\frac{\nu|\nabla g|_{\infty} \lambda_{m}}{2 m_{0} \lambda_{1}^{1 / 2}} e^{-\gamma \gamma_{0} \lambda_{1} s}\|\Phi\|^{2}+\frac{4}{\nu \gamma_{0}} e^{\nu \gamma_{0} \lambda_{1} s}|\xi|^{2} .
\end{aligned}
$$

Integrating this inequality from $s$ to $t$ and using (75), we have

$$
\begin{aligned}
e^{-\gamma \gamma_{0} \lambda_{1} s}\|\Phi\|^{2}+\frac{\nu \gamma_{0}}{4} \int_{s}^{t} e^{-\nu \gamma_{0} \lambda_{1} r}|A \Phi|^{2} d r \\
\leq c\left(1+\frac{\nu|\nabla g|_{\infty} \lambda_{m}}{2 m_{0} \lambda_{1}^{1 / 2}}+\sup _{t \geq 0}\left|u_{m}(t)\right|^{2}\left\|u_{m}(t)\right\|^{2}\right) \\
\quad \times e^{\left(2 / v \gamma_{0}\right) c_{0}^{2} \int_{0}^{t}\left\|u_{m}\right\|^{2} d r} \int_{0}^{t} e^{\nu \gamma_{0} \lambda_{1} r}|\xi|^{2} d r .
\end{aligned}
$$

Taking $v=\Phi_{s}$ in (67), then using (14), Lemmas 1 and 3, we obtain

$$
\left|\Phi_{s}\right| \leq \nu|A \Phi|+\nu \frac{|\nabla g|_{\infty} \lambda_{m}}{m_{0} \lambda_{1}^{1 / 2}}\|\Phi\|+c\left\|u_{m}\right\||A \Phi|+e^{\nu \gamma_{0} \lambda_{1} s}|\xi| .
$$

Hence,

$$
\begin{aligned}
e^{-\nu \gamma_{0} \lambda_{1} s}\left|\Phi_{s}\right|^{2} \leq & c\left(1+\left\|u_{m}\right\|^{2}\right) e^{-\gamma \gamma_{0} \lambda_{1} s}|A \Phi|^{2} \\
& +c e^{-\gamma \gamma_{0} \lambda_{1} s}\|\Phi\|^{2}+c e^{\nu \gamma_{0} \lambda_{1} s}|\xi|^{2} .
\end{aligned}
$$

Integrating (84) from $s$ to $t$ and using (82), (75), and Theorem 8 , we complete the proof. 
Lemma 10. If $u_{0} \in V_{g}$, then the error $P_{m} u(t)-u_{m}(t)$ satisfies

$$
\begin{gathered}
\left|P_{m} u(t)-u_{m}(t)\right|^{2}+\frac{v \gamma_{0}}{4} e^{-\gamma \gamma_{0} \lambda_{1} t} \int_{0}^{t} e^{\nu \gamma_{0} \lambda_{1} s}\left\|P_{m} u-u_{m}\right\|^{2} d s \\
\leq \mathscr{K} e^{\left(2 / v \gamma_{0}\right) c_{0}^{2} \int_{0}^{t}\|u(r)\|^{2} d r} \lambda_{m+1}^{-1} \quad \forall t \geq 0 .
\end{gathered}
$$

Proof. We set $e(t)=P_{m} u(t)-u_{m}(t)$ and subtract (66) from (62) to obtain

$e_{t}+\nu A e+\nu C e+P_{m} B\left(Q_{m} u+e, u\right)+P_{m} B\left(u_{m}, Q u_{m}+e\right)=0$,

with $e(0)=0$. Taking the scalar product of (86) with $2 e$, we obtain

$$
\begin{aligned}
\frac{d}{d t}|e|^{2} & +2 v\|e\|^{2}+2(C e, e)_{g} \\
& +2\left(b(e, u, e)+b\left(u_{m}, Q_{m} u, e\right)+b\left(Q_{m} u, u, e\right)\right)=0 .
\end{aligned}
$$

Using Lemma 3, we get

$$
\begin{aligned}
\frac{d}{d t}|e|^{2} & +2 \nu \gamma_{0}\|e\|^{2} \\
& \leq 2\left|b(e, u, e)+b\left(u_{m}, Q_{m} u, e\right)+b\left(Q_{m} u, u, e\right)\right| .
\end{aligned}
$$

Multiplying the last inequality by $e^{\nu \gamma_{0} \lambda_{1} t}$ and using (14), we obtain

$$
\begin{aligned}
& \frac{d}{d t}\left(e^{\nu \gamma_{0} \lambda_{1} t}|e|^{2}\right)+\nu \gamma_{0} e^{\nu \gamma_{0} \lambda_{1} t}\|e\|^{2} \\
& \quad \leq 2 e^{\nu \gamma_{0} \lambda_{1} t}\left|b(e, u, e)+b\left(u_{m}, Q_{m} u, e\right)+b\left(Q_{m} u, u, e\right)\right|
\end{aligned}
$$

Due to Lemma 1 and Cauchy's inequality, we have

$$
\begin{aligned}
2|b(e, u, e)| & \leq 2 c_{0}|e|\|e\|\|u\| \leq \frac{v \gamma_{0}}{2}\|e\|^{2}+\frac{2}{\nu \gamma_{0}} c_{0}^{2}\|u\|^{2}|e|^{2}, \\
2\left|b\left(u_{m}, Q_{m} u, e\right)\right|+2\left|b\left(Q_{m} u, u, e\right)\right| & \\
& \leq 2 c_{0} \lambda_{1}^{-1 / 2}\left|Q_{m} u\right|\left(|A u|+\left|A u_{m}\right|\right)\|e\| \\
& \leq \frac{v \gamma_{0}}{4}\|e\|^{2}+c\left(|A u|^{2}+\left|A u_{m}\right|^{2}\right)\left|Q_{m} u\right|^{2} .
\end{aligned}
$$

Combining (89) with the above estimate yields

$$
\begin{aligned}
& \frac{d}{d t}\left(e^{\nu \gamma_{0} \lambda_{1} t}|e|^{2}\right)+\frac{\gamma \gamma_{0}}{4} e^{\nu \gamma_{0} \lambda_{1} t}\|e\|^{2} \\
& \quad \leq \frac{2}{\nu \gamma_{0}} c_{0}^{2} e^{\nu \gamma_{0} \lambda_{1} t}\|u\|^{2}|e|^{2}+c e^{\nu \gamma_{0} \lambda_{1} t}\left(|A u|^{2}+\left|A u_{m}\right|^{2}\right)\left|Q_{m} u\right|^{2} .
\end{aligned}
$$

Multiplying (91) by $e^{-\left(2 / \nu \gamma_{0}\right) c_{0}^{2} \int_{0}^{s}\|u\|^{2} d r}$ yields

$$
\begin{aligned}
\frac{d}{d t}( & \left.e^{-\left(2 / \nu \gamma_{0}\right) c_{0}^{2} \int_{0}^{s}\|u\|^{2} d r} e^{\nu \gamma_{0} \lambda_{1} t}|e|^{2}\right) \\
& +\frac{\nu \gamma_{0}}{4} e^{-\left(2 / \nu \gamma_{0}\right) c_{0}^{2} \int_{0}^{s}\|u\|^{2} d r} e^{\nu \gamma_{0} \lambda_{1} t}\|e\|^{2} \\
\leq & c e^{-\left(2 / \nu \gamma_{0}\right) c_{0}^{2} \int_{0}^{s}\|u\|^{2} d r} e^{\nu \gamma_{0} \lambda_{1} t}\left(|A u|^{2}+\left|A u_{m}\right|^{2}\right)\left|Q_{m} u\right|^{2} \\
\leq & c e^{\nu \gamma_{0} \lambda_{1} t}\left(|A u|^{2}+\left|A u_{m}\right|^{2}\right)\left|Q_{m} u\right|^{2} .
\end{aligned}
$$

Integrating this inequality from 0 to $t$ and using (64), Theorem 8 , we obtain, after a final multiplication by $e^{\left(2 / \nu \gamma_{0}\right) c_{0}^{2} \int_{0}^{s}\|u\|^{2} d r} e^{-\gamma \gamma_{0} \lambda_{1} t}$, that

$$
\begin{aligned}
& |e|^{2}+\frac{\nu \gamma_{0}}{4} e^{-\nu \gamma_{0} \lambda_{1} t} \int_{0}^{t} e^{\nu \gamma_{0} \lambda_{1} s}\|e\|^{2} d s \\
& \leq \mathscr{K} e^{\left(2 / \nu \gamma_{0}\right) c_{0}^{2} \int_{0}^{t}\|u\|^{2} d r} \lambda_{m+1}^{-1} \quad \forall t \geq 0,
\end{aligned}
$$

which is (85).

Lemma 11. If $u_{0} \in V_{g}$, then the error $P_{m} u(t)-u_{m}(t)$ satisfies the following bound:

$$
\begin{aligned}
& \tau(t)\left|P_{m} u(t)-u_{m}(t)\right|^{2} \\
& \quad \leq \mathscr{K} e^{\left(4 / v \gamma_{0}\right) c_{0}^{2} \int_{0}^{t}\left(\|u\|^{2}+\left\|u_{m}\right\|^{2}\right) d r} \lambda_{m+1}^{-2} \quad \forall t \geq 0 .
\end{aligned}
$$

Proof. Take $v=e(s)$ and $\xi=e(s)$ in (67) to obtain

$$
\begin{gathered}
\left(e, \Phi_{s}\right)_{g}-a(e, \Phi)-\nu(C e, \Phi)_{g}-b\left(e, u_{m}, \Phi\right)-b\left(u_{m}, e, \phi\right) \\
=e^{\nu \gamma_{0} \lambda_{1} s}|e|^{2} .
\end{gathered}
$$

Multiplying (86) by $\Phi$, we have

$$
\begin{aligned}
& \left(e_{s}, \Phi\right)_{g}+a(e, \Phi)+v(C e, \Phi)_{g}+b\left(u_{m}, e+Q_{m} u, \Phi\right) \\
& +b\left(e+Q_{m} u, u_{m}, \Phi\right)+b\left(u-u_{m}, u-u_{m}, \Phi\right)=0 .
\end{aligned}
$$

Adding (96) and (95), we get

$$
\begin{aligned}
e^{\nu \gamma_{0} \lambda_{1} s}|e|^{2}= & \frac{d}{d s}(e, \Phi)_{g}+b\left(u_{m}, Q_{m} u, \Phi\right) \\
& +b\left(Q_{m} u, u_{m}, \Phi\right)+b\left(u-u_{m}, u-u_{m}, \Phi\right) .
\end{aligned}
$$

Using Lemma 1, we have

$$
\begin{gathered}
\left|b\left(u_{m}, Q_{m} u, \Phi\right)\right|+\left|b\left(Q_{m} u, u_{m}, \Phi\right)\right| \leq c\left\|u_{m}\right\|\left|Q_{m} u\right||A \Phi|, \\
\left|b\left(u-u_{m}, u-u_{m}, \Phi\right)\right| \leq c\left|u-u_{m}\right|\left|u-u_{m}\right|\|A \Phi\| .
\end{gathered}
$$

Hence, we deduce from (97) that

$$
\begin{aligned}
& e^{v \gamma_{0} \lambda_{1} s}|e|^{2} \\
& \quad \leq \frac{d}{d s}(e, \Phi)_{g}+c\left(\left\|u_{m}\right\|\left|Q_{m} u\right|+\left|u-u_{m}\right|\left\|u-u_{m}\right\|\right)|A \Phi| .
\end{aligned}
$$


Integrating (99) from 0 to $t$ and noting that $e(0)=\Phi(t)=0$, we obtain

$$
\begin{aligned}
& \int_{0}^{t} e^{\nu \gamma_{0} \lambda_{1} s}|e|^{2} d s \\
& \leq c\left(\int_{0}^{t} e^{\nu \gamma_{0} \lambda_{1} s}\left(\left\|u_{m}\right\|^{2}\left|Q_{m} u\right|^{2}+\left|u-u_{m}\right|^{2}\left\|u-u_{m}\right\|^{2}\right) d s\right)^{1 / 2} \\
& \quad \times\left(\int_{0}^{t} e^{-v \gamma_{0} \lambda_{1} s}|A \Phi|^{2} d s\right)^{1 / 2} .
\end{aligned}
$$

Using (64), (65), Theorem 8, and Lemmas 9 and 10, we deduce from (100) that

$$
e^{-\gamma \gamma_{0} \lambda_{1} t} \int_{0}^{t} e^{\nu \gamma_{0} \lambda_{1} s}|e|^{2} d s \leq \mathscr{K} e^{\left(4 / \nu \gamma_{0}\right) c_{0}^{2} \int_{0}^{t}\left(\|u\|^{2}+\left\|u_{m}\right\|^{2}\right) d r} \lambda_{m+1}^{-2} .
$$

Now, multiplying (91) by $\tau(t)$, we have

$$
\begin{aligned}
\frac{d}{d t}\left(\tau(t) e^{\nu \gamma_{0} \lambda_{1} t}|e|^{2}\right)+\frac{\nu \gamma_{0}}{4} \tau(t) e^{\nu \gamma_{0} \lambda_{1} t}\|e\|^{2} \\
\leq\left(1+\frac{2}{\nu \gamma_{0}} c_{0}^{2}\|u\|^{2}\right) e^{\nu \gamma_{0} \lambda_{1} t}|e|^{2} \\
\quad+c e^{\nu \gamma_{0} \lambda_{1} t} \tau(t)\left(|A u|^{2}+\left|A u_{m}\right|^{2}\right)\left|Q_{m} u\right|^{2} .
\end{aligned}
$$

Integrating (102) from 0 to $t$ and using Theorem 8 , we obtain, after a final multiplication by $e^{-\nu \gamma_{0} \lambda_{1} t}$,

$$
\begin{array}{r}
\tau(t)|e(t)|^{2}+\frac{\nu \gamma_{0}}{4} e^{-\nu \gamma_{0} \lambda_{1} t} \int_{0}^{t} \tau(s) e^{\nu \gamma_{0} \lambda_{1} s}\|e\|^{2} d s \\
\leq \mathscr{K} e^{-\nu \gamma_{0} \lambda_{1} t} \int_{0}^{t} e^{\nu \gamma_{0} \lambda_{1} t}\left(|e|^{2}+\left|Q_{m} u\right|^{2}\right) d s .
\end{array}
$$

Using (65) and (101) in (103) yields

$$
\begin{gathered}
\tau(t)|e(t)|^{2}+\frac{v \gamma_{0}}{4} e^{-v \gamma_{0} \lambda_{1} t} \int_{0}^{t} \tau(s) e^{\nu \gamma_{0} \lambda_{1} s}\|e\|^{2} d s \\
\leq \mathscr{K} e^{\left(4 / v \gamma_{0}\right) c_{0}^{2} \int_{0}^{t}\left(\|u\|^{2}+\left\|u_{m}\right\|^{2}\right) d r} \lambda_{m+1}^{-2},
\end{gathered}
$$

which is (94).

Finally, by combining Lemma 11 with (64) and using Theorem 8 , we get the following error estimate.

Theorem 12. If $u \in V_{g}$, then the error $u(t)-u_{m}(t)$ satisfies the following bound:

$$
\tau(t)\left|u(t)-u_{m}(t)\right|^{2} \leq \mathscr{K} e^{\left(8 / v^{3} \gamma_{0}^{3} \lambda_{1}\right) c_{0}^{2} C_{f}^{2} t} \lambda_{m+1}^{-2} \quad \forall t \geq 0 .
$$

\section{Spectral Galerkin Method in Space and Time}

5.1. Stability Analysis. In this subsection, we consider the semi-implicit Euler scheme applied to the spatially discrete spectral Galerkin approximation, show the stability of this scheme, and establish some preliminaries related to the error analysis uniform in time.

We consider the semi-implicit Euler scheme and define recursively a solution $\left\{u_{m}^{n}\right\} \subset H_{m}$ such that

$$
d_{t} u_{m}^{n+1}+v A u_{m}^{n+1}+v C u_{m}^{n+1}+P_{m} B\left(u_{m}^{n}, u_{m}^{n}\right)=P_{m} f^{n+1}
$$

for $n \geq 0$ with the initial condition $u_{m}^{0}=P_{m} u_{0}$, where $\Delta t>0$ is a time step such that $n_{0} \Delta t=1$ for some integer $n_{0}$ and

$$
\begin{gathered}
d_{t} u_{m}^{n+1}=\frac{1}{\Delta t}\left(u_{m}^{n+1}-u_{m}^{n}\right), \\
f^{n+1}=\frac{1}{\Delta t} \int_{t_{n}}^{t_{n+1}} f(t) d t, \quad t_{n}=n \Delta t .
\end{gathered}
$$

In order to derive the $L^{2}$-bound on the error $u_{m}\left(t_{n}\right)-u_{m}^{n}$, we will begin with a time discrete duality argument which is similar to the one used in $[11,17]$. We consider the dual scheme correponding to scheme (106): for any fixed $n \geq 1$ and $u_{m}^{k-1}, \xi^{k} \in H_{m}, 1 \leq k \leq n$, find $\Phi^{k-1} \in H_{m}, 1 \leq k \leq n$ such that

$$
\begin{gathered}
\left(v, d_{t} \Phi^{k}\right)_{g}-a\left(v, \Phi^{k-1}\right)-v\left(C v, \Phi^{k-1}\right)_{g}-b\left(v, u_{m}^{k-1}, \Phi^{k-1}\right) \\
-b\left(u_{m}^{k-1}, v, \Phi^{k-1}\right)=\left(1+v \gamma_{0} \lambda_{1} \Delta t\right)^{k}\left(v, \xi^{k}\right)_{g}
\end{gathered}
$$$$
\forall v \in H_{m}
$$

with an initial condition $\Phi^{n}=0$.

The following theorem provides the $H^{2}$-stability of scheme (106).

Theorem 13. Under the assumptions of Theorem 8, if $\Delta t$ and $m$ satisfy the following condition:

$$
\frac{8}{v \gamma_{0}} c_{0}^{2} \ln \frac{\lambda_{m}}{\lambda_{1}} \Delta t C_{u_{0}, f} \leq 1
$$

then the semi-implicit Euler scheme is the $H^{2}$-stability; that is,

$$
\Delta t \sum_{k=1}^{n}\left\|u_{m}^{k}\right\|^{2} \leq \frac{1}{\nu \gamma_{0}}\left|u_{0}\right|^{2}+\frac{1}{v^{2} \gamma_{0}^{2} \lambda_{1}} C_{f}^{2} t_{n} \quad \forall n \geq 0,
$$

$$
\begin{array}{r}
\Delta t \sum_{k=k_{0}+1}^{n}\left\|u_{m}^{k}\right\|^{2} \\
\leq \frac{1}{v \gamma_{0}}\left|u_{0}\right|^{2}+\frac{2}{\nu^{3} \gamma_{0}^{3} \lambda_{1}^{2}} C_{f}^{2}+\frac{2}{v^{2} \gamma_{0}^{2} \lambda_{1}} C_{f}^{2}\left(t_{n}-t_{k_{0}}\right) \\
\forall n \geq k_{0} \geq 0,
\end{array}
$$




$$
\begin{aligned}
& \left|u_{m}^{n}\right|^{2} \leq\left(1+v \gamma_{0} \lambda_{1} \Delta t\right)^{-n}\left|u_{0}\right|^{2}+\frac{1}{v^{2} \gamma_{0}^{2} \lambda_{1}^{2}} C_{f}^{2} \quad \forall n \geq 0, \\
& \left\|u_{m}^{n}\right\|^{2} \leq C_{u_{0}, f} \quad \forall n \geq 0 \\
& \Delta t \sum_{k=1}^{n}\left(1+v \gamma_{0} \lambda_{1} \Delta t\right)^{-(n-1-k)} \\
& \quad \times\left(\left|A u_{m}^{k}\right|^{2}+\left|d_{t} u_{m}^{k}\right|^{2}+\tau\left(t_{k}\right)\left\|d_{t} u_{m}^{k}\right\|^{2}\right) \\
& \quad+\tau\left(t_{n}\right)\left|A u_{m}^{n}\right|^{2}+\tau\left(t_{n}\right)\left|d_{t} u_{m}^{n}\right|^{2} \leq \mathscr{K} \quad \forall n \geq 0 .
\end{aligned}
$$

Proof. Clearly, scheme (106) defines a unique sequence $\left\{u_{m}^{n}\right\} \subset H_{m}$. Now, we will prove (111)-(115).

Taking the scalar product of (106) with $2 u_{m}^{n+1} \Delta t$, we obtain

$$
\begin{aligned}
\left|u_{m}^{n+1}\right|^{2} & -\left|u_{m}^{n}\right|^{2}+\left|u_{m}^{n+1}-u_{m}^{n}\right|^{2}+2 v\left\|u_{m}^{n+1}\right\|^{2} \Delta t \\
& +2 \nu \Delta t\left(C u_{m}^{n+1}, u_{m}^{n+1}\right)_{g}+2 b\left(u_{m}^{n}, u_{m}^{n}-u_{m}^{n+1}, u_{m}^{n+1}\right) \Delta t \\
= & 2\left(f^{n+1}, u_{m}^{n+1}\right)_{g} \Delta t
\end{aligned}
$$

Using Lemma 3 and Cauchy's inequality, we have

$$
\begin{aligned}
\left|u_{m}^{n+1}\right|^{2}-\left|u_{m}^{n}\right|^{2}+\left|u_{m}^{n+1}-u_{m}^{n}\right|^{2}+2 v\left\|u_{m}^{n+1}\right\|^{2} \Delta t \\
\leq 2 v \frac{|\nabla g|_{\infty}}{m_{0} \lambda_{1}^{1 / 2}}\left\|u_{m}^{n+1}\right\|^{2} \Delta t+2\left(f^{n+1}, u_{m}^{n+1}\right)_{g} \Delta t \\
-2 b\left(u_{m}^{n}, u_{m}^{n}-u_{m}^{n+1}, u_{m}^{n+1}\right) \Delta t .
\end{aligned}
$$

Hence,

$$
\begin{aligned}
& \left|u_{m}^{n+1}\right|^{2}-\left|u_{m}^{n}\right|^{2}+\left|u_{m}^{n+1}-u_{m}^{n}\right|^{2}+2 \nu \gamma_{0}\left\|u_{m}^{n+1}\right\|^{2} \Delta t \\
& \quad \leq 2\left(f^{n+1}, u_{m}^{n+1}\right)_{g} \Delta t-2 b\left(u_{m}^{n}, u_{m}^{n}-u_{m}^{n+1}, u_{m}^{n+1}\right) \Delta t
\end{aligned}
$$

By (19), we have

$$
\begin{gathered}
2\left(f^{n+1}, u_{m}^{n+1}\right)_{g} \leq \frac{v \gamma_{0}}{2}\left\|u_{m}^{n+1}\right\|^{2}+\frac{2}{\nu \gamma_{0} \lambda_{1}}\left|f^{n+1}\right|^{2} \Delta t, \\
2\left|b\left(u_{m}^{n}, u_{m}^{n}-u_{m}^{n+1}, u_{m}^{n+1}\right)\right| \\
\leq \frac{\nu \gamma_{0}}{2}\left\|u_{m}^{n+1}\right\|^{2}+L\left(u_{m}^{n}\right)\left|u_{m}^{n}-u_{m}^{n+1}\right|^{2},
\end{gathered}
$$

where

$$
L\left(u_{m}^{n}\right)=\frac{8}{v \gamma_{0}} c_{0}^{2}\left(1+\ln \frac{\left|A u_{m}^{n}\right|^{2}}{\lambda_{1}\left\|u_{m}^{n}\right\|^{2}}\right)\left\|u_{m}^{n}\right\|^{2} .
$$

Substituting those into (118), we get

$$
\begin{gathered}
\left|u_{m}^{n+1}\right|^{2}-\left|u_{m}^{n}\right|^{2}+\left(1-L\left(u_{m}^{n}\right) \Delta t\right)\left|u_{m}^{n+1}-u_{m}^{n}\right|^{2} \\
+v \gamma_{0}\left\|u_{m}^{n+1}\right\|^{2} \Delta t \leq \frac{2}{v \gamma_{0} \lambda_{1}}\left|f^{n+1}\right|^{2} \Delta t .
\end{gathered}
$$

Next, by taking the scalar product of (106) with $2 A u_{m}^{n+1} \Delta t$, we obtain

$$
\begin{aligned}
& \left\|u_{m}^{n+1}\right\|^{2}-\left\|u_{m}^{n}\right\|^{2}+\left\|u_{m}^{n+1}-u_{m}^{n}\right\|^{2}+2 \nu\left|A u_{m}^{n+1}\right|^{2} \Delta t \\
& \quad+2 v \Delta t\left(C u_{m}^{n+1}, A u_{m}^{n+1}\right)_{g}+2 b\left(u_{m}^{n}, u_{m}^{n}, A u_{m}^{n+1}\right) \Delta t \\
& \quad=2\left(f^{n+1}, A u_{m}^{n+1}\right)_{g} \Delta t .
\end{aligned}
$$

Using Lemma 3 and Cauchy's inequality, we have

$$
\begin{aligned}
& 2 \nu\left(C u_{m}^{n+1}, A u_{m}^{n+1}\right)_{g} \\
& \quad \leq 2 \nu \frac{|\nabla g|_{\infty}}{m_{0} \lambda_{1}^{1 / 2}}\left|A u_{m}^{n+1}\right|^{2}+\frac{\nu|\nabla g|_{\infty} \lambda_{1}^{1 / 2}}{2 m_{0}}\left\|u_{m}^{n+1}\right\|^{2}
\end{aligned}
$$

Substituting into (122), we get

$$
\begin{gathered}
\left\|u_{m}^{n+1}\right\|^{2}-\left\|u_{m}^{n}\right\|^{2}+\left\|u_{m}^{n+1}-u_{m}^{n}\right\|^{2}+2 \nu \gamma_{0}\left|A u_{m}^{n+1}\right|^{2} \Delta t \\
\leq 2\left(f^{n+1}, A u_{m}^{n+1}\right)_{g} \Delta t-2 b\left(u_{m}^{n}, u_{m}^{n}, A u_{m}^{n+1}\right) \Delta t \\
\quad+\frac{\nu|\nabla g|_{\infty} \lambda_{1}^{1 / 2}}{2 m_{0}}\left\|u_{m}^{n+1}\right\|^{2} \Delta t .
\end{gathered}
$$

Due to (17)-(19), Cauchy's inequality, and Young's inequality, we have

$$
\begin{aligned}
& 2\left|b\left(u_{m}^{n}, u_{m}^{n+1}-u_{m}^{n}, A u_{m}^{n+1}\right)\right| \\
& \quad \leq \frac{\nu \gamma_{0}}{4}\left|A u_{m}^{n+1}\right|^{2}+L\left(u_{m}^{n}\right)\left\|u_{m}^{n+1}-u_{m}^{n}\right\|^{2},
\end{aligned}
$$

$$
\begin{aligned}
& 2\left|b\left(u_{m}^{n}, u_{m}^{n+1}, A u_{m}^{n+1}\right)\right| \\
& \leq \nu \gamma_{0}\left|A u_{m}^{n+1}\right|^{2}+2\left(\nu \gamma_{0}\right)^{-3} c_{0}^{4}\left|u_{m}^{n}\right|^{2}\left\|u_{m}^{n}\right\|^{2}\left\|u_{m}^{n+1}\right\|^{2} \\
& 2\left(f^{n+1}, A u_{m}^{n+1}\right)_{g} \leq \frac{\nu \gamma_{0}}{2}\left|A u_{m}^{n+1}\right|^{2}+\frac{2}{\nu \gamma_{0}} C_{f}^{2} .
\end{aligned}
$$

By combining (124) with the above estimates, we obtain

$$
\begin{aligned}
\left\|u_{m}^{n+1}\right\|^{2}-\left\|u_{m}^{n}\right\|^{2}+\left(1-L\left(u_{m}^{n}\right) \Delta t\right)\left\|u_{m}^{n+1}-u_{m}^{n}\right\|^{2} \\
\leq 2\left(\nu \gamma_{0}\right)^{-3} c_{0}^{4}\left|u_{m}^{n}\right|^{2}\left\|u_{m}^{n}\right\|^{2}\left\|u_{m}^{n+1}\right\|^{2} \Delta t+\frac{2}{v \gamma_{0}} C_{f}^{2} \Delta t \\
+\frac{\nu|\nabla g|_{\infty} \lambda_{1}^{1 / 2}}{2 m_{0}}\left\|u_{m}^{n+1}\right\|^{2} \Delta t .
\end{aligned}
$$


On the other hand, from (17)-(19), Cauchy's inequality, and Young's inequality, we have

$$
\begin{aligned}
& 2\left|b\left(u_{m}^{n}, u_{m}^{n+1}-u_{m}^{n}, A u_{m}^{n+1}\right)\right| \\
& \quad \leq \frac{v \gamma_{0}}{4}\left|A u_{m}^{n+1}\right|^{2}+L\left(u_{m}^{n}\right)\left\|u_{m}^{n+1}-u_{m}^{n}\right\|^{2},
\end{aligned}
$$

$2\left|b\left(u_{m}^{n}, u_{m}^{n+1}, A u_{m}^{n+1}\right)\right| \leq \frac{\nu \gamma_{0}}{8}\left|A u_{m}^{n+1}\right|^{2}+c\left|u_{m}^{n}\right|^{2}\left\|u_{m}^{n}\right\|^{2}\left\|u_{m}^{n+1}\right\|^{2}$

$$
2\left(f^{n+1}, A u_{m}^{n+1}\right)_{g} \leq \frac{\nu \gamma_{0}}{8}\left|A u_{m}^{n+1}\right|^{2}+c C_{f}^{2} \text {. }
$$

Combining (124) with those estimates, we obtain

$$
\begin{aligned}
& \left(1+v \gamma_{0} \lambda_{1} \Delta t\right)\left\|u_{m}^{n+1}\right\|^{2}-\left\|u_{m}^{n}\right\|^{2} \\
& \quad+\left(1-L\left(u_{m}^{n}\right) \Delta t\right)\left\|u_{m}^{n+1}-u_{m}^{n}\right\|^{2}+\frac{\nu \gamma_{0}}{2}\left|A u_{m}^{n+1}\right|^{2} \Delta t \\
& \leq c\left|u_{m}^{n}\right|^{2}\left\|u_{m}^{n}\right\|^{2}\left\|u_{m}^{n+1}\right\|^{2} \Delta t+c C_{f}^{2} \Delta t+c\left\|u_{m}^{n+1}\right\|^{2} \Delta t .
\end{aligned}
$$

Next, from (106) we have

$$
\begin{gathered}
\left|d_{t} u_{m}^{n+1}\right|^{2}+v\left(A u_{m}^{n+1}, d_{t} u_{m}^{n+1}\right)+v\left(C u_{m}^{n+1}, d_{t} u_{m}^{n+1}\right)_{g} \\
+b\left(u^{n}, u^{n}, d_{t} u_{m}^{n+1}\right)=\left(f^{n+1}, d_{t} u_{m}^{n+1}\right)_{g} .
\end{gathered}
$$

Using (11), Lemmas 1 and 3, and Cauchy's inequality, we get

$$
\begin{gathered}
\left|b\left(u^{n}, u^{n}, d_{t} u_{m}^{n+1}\right)\right| \leq c\left|u_{m}^{n}\right|^{2}\left\|u_{m}^{n}\right\|\left\|u_{m}^{n+1}\right\|^{2}, \\
\nu\left|\left(A u_{m}^{n+1}, d_{t} u_{m}^{n+1}\right)_{g}\right| \leq \frac{1}{4}\left|d_{t} u_{m}^{n+1}\right|^{2}+v^{2}\left|A u_{m}^{n+1}\right|^{2}, \\
\nu\left|\left(C u_{m}^{n+1}, d_{t} u_{m}^{n+1}\right)_{g}\right| \leq \frac{1}{4}\left|d_{t} u_{m}^{n+1}\right|^{2}+c\left\|u_{m}^{n+1}\right\|^{2}, \\
\left|\left(f^{n+1}, d_{t} u_{m}^{n+1}\right)_{g}\right| \leq \frac{1}{4}\left|d_{t} u_{m}^{n+1}\right|^{2}+c C_{f}^{2} .
\end{gathered}
$$

Therefore, we have

$$
\begin{aligned}
& \frac{\gamma_{0}}{16 v}\left|d_{t} u_{m}^{n+1}\right|^{2}-\frac{v \gamma_{0}}{4}\left|A u_{m}^{n+1}\right|^{2} \\
& \quad \leq c\left|u_{m}^{n}\right|^{2}\left\|u_{m}^{n}\right\|^{2}\left\|u_{m}^{n+1}\right\|^{2}+c C_{f}^{2}+c\left\|u_{m}^{n+1}\right\|^{2} .
\end{aligned}
$$

Combining this inequality with (128) yields

$$
\begin{aligned}
(1+ & \left.\nu \gamma_{0} \lambda_{1} \Delta t\right)\left\|u_{m}^{n+1}\right\|^{2} \\
& -\left\|u_{m}^{n}\right\|^{2}+\left(1-L\left(u_{m}^{n}\right) \Delta t\right)\left\|u_{m}^{n+1}-u_{m}^{n}\right\|^{2} \\
& +\frac{\gamma_{0}}{16 \nu}\left|d_{t} u_{m}^{n+1}\right|^{2} \Delta t+\frac{\nu \gamma_{0}}{4}\left|A u_{m}^{n+1}\right|^{2} \Delta t \\
\leq & c\left|u_{m}^{n}\right|^{2}\left\|u_{m}^{n}\right\|^{2}\left\|u_{m}^{n+1}\right\|^{2} \Delta t+c C_{f}^{2} \Delta t+c\left\|u_{m}^{n+1}\right\|^{2} \Delta t .
\end{aligned}
$$

Moreover, we deduce from (106) that

$$
\begin{gathered}
d_{t t} u_{m}^{n+1}+v A d_{t} u_{m}^{n+1}+v C d_{t} u_{m}^{n+1}+P_{m} B\left(d_{t} u_{m}^{n}, u_{m}^{n}\right) \\
+P_{m} B\left(u_{m}^{n}, d_{t} u_{m}^{n}\right)=P_{m} d_{t} f^{n+1}
\end{gathered}
$$

where $d_{t} u_{m}^{0}$ is defined by

$$
d_{t} u_{m}^{0}+v A u_{m}^{0}+v C u_{m}^{0}+P_{m} B\left(u_{m}^{0}, u_{m}^{0}\right)=P_{m} f(0) .
$$

Taking the scalar product of (133) with $2 d_{t} u_{m}^{n+1} \Delta t$, we obtain

$$
\begin{aligned}
\mid d_{t} u_{m}^{n+1} & \left.\right|^{2}-\left|d_{t} u_{m}^{n}\right|^{2}+\left|d_{t} u_{m}^{n+1}-d_{t} u_{m}^{n}\right|^{2} \\
& +2 v\left\|d_{t} u_{m}^{n+1}\right\|^{2} \Delta t+2 v\left(C d_{t} u_{m}^{n+1}, d_{t} u_{m}^{n+1}\right) \Delta t \\
& +2 b\left(d_{t} u_{m}^{n}-d_{t} u_{m}^{n+1}, u_{m}^{n}, d_{t} u_{m}^{n+1}\right) \Delta t \\
& +2 b\left(d_{t} u_{m}^{n+1}, u_{m}^{n}, d_{t} u_{m}^{n+1}\right) \Delta t \\
& +2 b\left(d_{t} u_{m}^{n+1}, d_{t} u_{m}^{n}-d_{t} u_{m}^{n+1}, d_{t} u_{m}^{n+1}\right) \Delta t \\
= & 2\left(d_{t} f^{n+1}, d_{t} u_{m}^{n+1}\right) \Delta t .
\end{aligned}
$$

By Lemma 3, we get

$$
\begin{aligned}
\left|d_{t} u_{m}^{n+1}\right|^{2}-\left|d_{t} u_{m}^{n}\right|^{2}+\left|d_{t} u_{m}^{n+1}-d_{t} u_{m}^{n}\right|^{2}+2 v \gamma_{0}\left\|d_{t} u_{m}^{n+1}\right\|^{2} \Delta t \\
\leq 2\left(d_{t} f^{n+1}, d_{t} u_{m}^{n+1}\right) \Delta t \\
\quad-2 b\left(d_{t} u_{m}^{n}-d_{t} u_{m}^{n+1}, u_{m}^{n}, d_{t} u_{m}^{n+1}\right) \Delta t \\
\quad-2 b\left(d_{t} u_{m}^{n+1}, u_{m}^{n}, d_{t} u_{m}^{n+1}\right) \Delta t \\
\quad-2 b\left(d_{t} u_{m}^{n+1}, d_{t} u_{m}^{n}-d_{t} u_{m}^{n+1}, d_{t} u_{m}^{n+1}\right) \Delta t
\end{aligned}
$$

Using Lemma 1 and (19), we have

$$
\begin{aligned}
& 2\left|b\left(d_{t} u_{m}^{n}-d_{t} u_{m}^{n+1}, u_{m}^{n}, d_{t} u_{m}^{n+1}\right)\right| \\
& \quad+2\left|b\left(d_{t} u_{m}^{n+1}, d_{t} u_{m}^{n}-d_{t} u_{m}^{n+1}, d_{t} u_{m}^{n+1}\right)\right| \\
& \leq \frac{v \gamma_{0}}{2}\left\|d_{t} u_{m}^{n+1}\right\|^{2}+\frac{1}{2}\left(L\left(u_{m}^{n}\right)+L\left(u_{m}^{n+1}\right)\right)\left|d_{t} u_{m}^{n+1}-d_{t} u_{m}^{n}\right|^{2}, \\
& 2\left|b\left(d_{t} u_{m}^{n+1}, u_{m}^{n}, d_{t} u_{m}^{n+1}\right)\right| \leq \frac{\nu \gamma_{0}}{8}\left\|d_{t} u_{m}^{n+1}\right\|^{2} \\
& \quad+\frac{8}{\nu \gamma_{0}} c_{0}^{2}\left\|u_{m}^{n}\right\|^{2}\left|d_{t} u_{m}^{n+1}\right|^{2}, \\
& 2\left(d_{t} f^{n+1}, d_{t} u_{m}^{n+1}\right) \leq \frac{\nu \gamma_{0}}{8}\left\|d_{t} u_{m}^{n+1}\right\|^{2}+\frac{8}{\nu \gamma_{0} \lambda_{1}} \sup _{t \geq 0}\left|f_{t}(t)\right|^{2} .
\end{aligned}
$$


By combining (136) with the above estimates, we arrive at

$$
\begin{aligned}
& \left(1+v \gamma_{0} \lambda_{1} \Delta t\right)\left|d_{t} u_{m}^{n+1}\right|^{2}-\left|d_{t} u_{m}^{n}\right|^{2} \\
& +\left(1-\frac{1}{2}\left(L\left(u_{m}^{n}\right)+L\left(u_{m}^{n+1}\right)\right) \Delta t\right)\left|d_{t} u_{m}^{n+1}-d_{t} u_{m}^{n}\right|^{2} \\
& +\frac{v \gamma_{0}}{4}\left\|d_{t} u_{m}^{n+1}\right\|^{2} \Delta t \leq \frac{8}{v \gamma_{0}} c_{0}^{2}\left\|u_{m}^{n}\right\|^{2}\left|d_{t} u_{m}^{n+1}\right|^{2} \Delta t \\
& +\frac{8}{v \gamma_{0} \lambda_{1}} \sup _{t \geq 0}\left|f_{t}(t)\right|^{2}
\end{aligned}
$$

Multiplying (138) by $\tau\left(t_{n+1}\right)$ and noting that $\tau\left(t_{n+1}\right) \leq \tau\left(t_{n}\right)+$ $\Delta t$, we obtain

$$
\begin{aligned}
(1+ & \left.\nu \gamma_{0} \lambda_{1} \Delta t\right) \tau\left(t_{n+1}\right)\left|d_{t} u_{m}^{n+1}\right|^{2}-\tau\left(t_{n}\right)\left|d_{t} u_{m}^{n}\right|^{2} \\
& +\left(1-\frac{1}{2}\left(L\left(u_{m}^{n}\right)+L\left(u_{m}^{n+1}\right)\right) \Delta t\right) \\
& \times \tau\left(t_{n+1}\right)\left|d_{t} u_{m}^{n+1}-d_{t} u_{m}^{n}\right|^{2} \\
& +\frac{\nu \gamma_{0}}{4} \tau\left(t_{n+1}\right)\left\|d_{t} u_{m}^{n+1}\right\|^{2} \Delta t \\
\leq & \left|d_{t} u_{m}^{n}\right|^{2} \Delta t+\frac{8}{\nu \gamma_{0}} c_{0}^{2}\left\|u_{m}^{n}\right\|^{2}\left|d_{t} u_{m}^{n+1}\right|^{2} \Delta t \\
& +\frac{8}{\nu \gamma_{0} \lambda_{1}} \sup _{t \geq 0}\left|f_{t}(t)\right|^{2} \Delta t .
\end{aligned}
$$

Now, we will prove (111)-(115) by induction. Obviously, (111)(115) are true for $n=0$. Assuming that (111)-(115) hold for $n=0,1, \ldots, J$, we need to prove (111)-(115) for $n=J+1$. In view of (110) and the inductive assumption, we obtain

$$
1-L\left(u_{m}^{n}\right) \Delta t \geq 0 \quad \forall 0 \leq n \leq J .
$$

Summing (121) from 0 to $J$ and $k_{0}+1$ to $J$, respectively, and using (140), we obtain (111) and (112) with $n=J+1$ after a final multiplication by $\left(v \gamma_{0}\right)^{-1}$. Noting that $\lambda_{1}|v|^{2} \leq\|v\|^{2}$, using (140) in (121), and then applying Lemma 5 with

$$
\begin{array}{cc}
a_{k}=\left|u_{m}^{k}\right|^{2}, & b_{k}=0, \\
h_{k}=\frac{2}{\nu \gamma_{0} \lambda_{1}} C_{f}^{2}, & \gamma=v \gamma_{0} \lambda_{1},
\end{array}
$$

we obtain (113) for $n=J+1$.

Furthermore, setting

$$
\begin{gathered}
a_{k}=\left\|u_{m}^{k}\right\|^{2}, \quad g_{k}=2\left(\nu \gamma_{0}\right)^{-3} c_{0}^{4}\left|u_{m}^{k}\right|^{2}\left\|u_{m}^{k+1}\right\|^{2}, \\
h_{k}=\frac{2}{\nu \gamma_{0}} C_{f}^{2}+\frac{\nu|\nabla g|_{\infty} \lambda_{1}^{1 / 2}}{2 m_{0}}\left\|u_{m}^{k+1}\right\|^{2},
\end{gathered}
$$

in (126), using (111)-(113) and (140), we obtain

$$
\frac{1}{\Delta t}\left(a_{k+1}-a_{k}\right) \leq g_{k} a_{k}+h_{k} \quad \forall 0 \leq k \leq J,
$$

with

$$
\Delta t \sum_{k=0}^{J} g_{k} \leq \alpha_{1}, \quad \Delta t \sum_{k=0}^{J} h_{k} \leq \alpha_{1}, \quad a_{0} \leq\left\|u_{0}\right\|^{2},
$$

for all $J+1 \leq n_{0}-1$ and

$$
\begin{gathered}
\Delta t \sum_{k=k_{0}}^{k_{0}+n_{0}-1} g_{k} \leq \alpha_{1}, \quad \Delta t \sum_{k=k_{0}}^{k_{0}+n_{0}-1} h_{k} \leq \alpha_{2}, \\
\Delta t \sum_{k=k_{0}}^{k_{0}+n_{0}-1} a_{k} \leq a_{0}+\alpha_{3}, \quad 0 \leq k_{0} \leq J+1-n_{0},
\end{gathered}
$$

for all $J+1 \geq n_{0}$. Applying Lemma 4 to (143), we obtain (114) for $n=J+1$.

Applying again Lemma 4 to (132) with

$$
\begin{gathered}
a_{k}=\left\|u_{m}^{k}\right\|^{2}, \\
b_{k}=\frac{1}{16 \nu \gamma_{0}}\left|d_{t} u_{m}^{k}\right|^{2}+\frac{\nu \gamma_{0}}{4}\left|A u_{m}^{k}\right|^{2}, \quad \gamma=v \gamma_{0} \lambda_{1}, \\
h_{k}=c\left|u_{m}^{k}\right|^{2}\left\|u_{m}^{k}\right\|^{2}\left\|u_{m}^{k+1}\right\|^{2}+c C_{f}^{2}+c\left\|u_{m}^{k+1}\right\|^{2},
\end{gathered}
$$

and using (111)-(114) and (140), we obtain

$$
\Delta t \sum_{k=1}^{J+1}\left(1+v \gamma_{0} \lambda_{1} \Delta t\right)^{-(J+2-k)}\left(\left|A u_{m}^{k}\right|+\left|d_{t} u_{m}^{k}\right|^{2}\right) \leq \mathscr{K}
$$

Applying Lemma 5 to (139) with

$$
\begin{aligned}
& a_{k}=\tau\left(t_{k}\right)\left|d_{t} u_{m}^{k}\right|^{2}, \quad b_{k}=\frac{v \gamma_{0}}{4} \tau\left(t_{k}\right)\left\|d_{t} u_{m}^{k}\right\|^{2}, \quad \gamma=v \gamma_{0} \lambda_{1}, \\
& h_{k}=\left|d_{t} u_{m}^{k}\right|^{2} \Delta t+\frac{8}{\nu \gamma_{0}} c_{0}^{2}\left\|u_{m}^{k}\right\|^{2}\left|d_{t} u_{m}^{k+1}\right|^{2}+\frac{8}{\nu \gamma_{0} \lambda_{1}} \sup _{t \geq 0}\left|f_{t}(t)\right|^{2},
\end{aligned}
$$

and using (114), (140), and (147) yields

$$
\begin{aligned}
& \tau\left(t_{J+1}\right)\left|d_{t} u_{m}^{J+1}\right|^{2} \\
& \quad+\frac{\nu \gamma_{0}}{4} \Delta t \sum_{k=1}^{J+1}\left(1+\nu \gamma_{0} \lambda_{1} \Delta t\right)^{-(J+2-k)} \tau\left(t_{k}\right)\left\|d_{t} u_{m}^{k}\right\|^{2} \leq \mathscr{K} .
\end{aligned}
$$

Finally, due to (17)-(19), we have

$$
\begin{aligned}
\left|b\left(u_{m}^{J}, u^{J}-u_{m}^{J+1}, A u_{m}^{J+1}\right)\right| \leq & \frac{\nu \gamma_{0}}{8}\left|A u_{m}^{J+1}\right|^{2} \\
& +c L\left(u_{m}^{J}\right)\left\|d_{t} u_{m}^{J+1}\right\|^{2} \Delta t^{2} \\
\left|b\left(u_{m}^{J}, u_{m}^{J+1}, A u_{m}^{J+1}\right)\right| \leq & \frac{\nu \gamma_{0}}{8}\left|A u_{m}^{J+1}\right|^{2} \\
& +c\left|u_{m}^{J}\right|^{2}\left\|u_{m}^{J}\right\|^{2}\left\|u_{m}^{J+1}\right\|^{2}
\end{aligned}
$$


Combining these inequalities with (106) and (140) yields

$$
\begin{aligned}
\tau t_{J+1}\left|A u_{m}^{J+1}\right|^{2} \leq & c \tau\left(t_{J+1}\right)\left|d_{t} u_{m}^{J+1}\right|^{2} \\
& +c\left|u_{m}^{J}\right|^{2}\left\|u_{m}^{J}\right\|\left\|^{2}\right\| u_{m}^{J+1} \|^{2} \\
& +c C_{f}^{2} \\
& +c \tau\left(t_{J+1}\right)\left\|d_{t} u_{m}^{J+1}\right\|^{2} \Delta t .
\end{aligned}
$$

Using (113), (114) and (149) in this inequality yields

$$
\tau\left(t_{J+1}\right)\left|A u_{m}^{J+1}\right|^{2} \leq \mathscr{K}
$$

Combining this inequality with (149) and (147) implies (115) for $n=J+1$. (109).

The following lemma provides the stability of scheme

Lemma 14. If $u_{0} \in V_{g}, \Delta t$ and $m$ satisfy (110), then problem (109) admits a unique solution $\left\{\Phi^{k}\right\}_{0}^{n} \subset H_{m}$ satisfying

$$
\begin{aligned}
& \left(1+\nu \gamma_{0} \lambda_{1} \Delta t\right)^{-r}\left\|\Phi^{r}\right\|^{2}+\Delta t \sum_{k=r}^{n}\left(1+\nu \gamma_{0} \lambda_{1} \Delta t\right)^{-(k-1)}\left|A \Phi^{k}\right|^{2} \\
& \quad \leq \mathscr{K} \exp \left(\frac{4}{\nu \gamma_{0}} c_{0}^{2} \Delta t \sum_{k=1}^{n}\left\|u_{m}^{k}\right\|^{2}\right) \Delta t \sum_{k=1}^{n}\left(1+\nu \gamma_{0} \lambda_{1} \Delta t\right)^{k}\left|\xi^{k}\right|^{2} .
\end{aligned}
$$

Proof. In view of (17), (110), and (114), we can prove that the following bilinear form

$$
\begin{gathered}
\frac{1}{\Delta t}(v, \Phi)+a(v, \Phi)+v(C v, \Phi)+b\left(v, u_{m}^{k-1}, \Phi\right) \\
+b\left(u_{m}^{k-1}, v, \Phi\right) \quad \forall v, \Phi \in H_{m}
\end{gathered}
$$

is elliptic. Hence, problem (109) has a unique solution $\Phi^{k-1}$ for $1 \leq k \leq n$. Next, by taking $v=-2\left(1+\nu \gamma_{0} \lambda_{1} \Delta t\right)^{-k} \Phi^{k-1} \Delta t$ in (109) and using (11), we have

$$
\begin{aligned}
-2(1 & \left.+\nu \gamma_{0} \lambda_{1} \Delta t\right)^{-k}\left(\Phi^{k-1}, \Phi^{k}\right)_{g} \\
& +2\left(1+\nu \gamma_{0} \lambda_{1} \Delta t\right)^{-k}\left|\Phi^{k-1}\right|^{2} \\
& +2 \nu\left(1+\nu \gamma_{0} \lambda_{1} \Delta t\right)^{-k}\left\|\Phi^{k-1}\right\|^{2} \Delta t \\
& +2 \nu\left(1+\nu \gamma_{0} \lambda_{1} \Delta t\right)^{-k}\left(C \Phi^{k-1}, \Phi^{k-1}\right)_{g} \Delta t \\
& +2\left(1+\nu \gamma_{0} \lambda_{1} \Delta t\right)^{-k} b\left(\Phi^{k-1}, u_{m}^{k-1}, \Phi^{k-1}\right) \Delta t \\
= & -2\left(\Phi^{k-1}, \xi^{k}\right)_{g} \Delta t .
\end{aligned}
$$

Using Lemma 3, we get

$$
\begin{aligned}
2(1+ & \left.\nu \gamma_{0} \lambda_{1} \Delta t\right)^{-k}\left|\Phi^{k-1}\right|^{2} \\
& +2 \nu \gamma_{0}\left(1+\nu \gamma_{0} \lambda_{1} \Delta t\right)^{-k}\left\|\Phi^{k-1}\right\|^{2} \Delta t \\
\leq & 2\left|\left(\Phi^{k-1}, \xi^{k}\right)_{g}\right| \Delta t+2\left(1+\nu \gamma_{0} \lambda_{1} \Delta t\right)^{-k}\left|\left(\Phi^{k-1}, \Phi^{k}\right)_{g}\right| \\
& +2\left(1+\nu \gamma_{0} \lambda_{1} \Delta t\right)^{-k}\left|b\left(\Phi^{k-1}, u_{m}^{k-1}, \Phi^{k-1}\right)\right| \Delta t .
\end{aligned}
$$

Using (17) and Cauchy's inequality, we have

$$
\begin{aligned}
2\left|\left(\Phi^{k-1}, \Phi^{k}\right)_{g}\right| & \leq\left|\Phi^{k-1}\right|^{2}+\left|\Phi^{k}\right|^{2}, \\
2\left|\left(\Phi^{k-1}, \xi^{k}\right)_{g}\right| \leq & \frac{\nu \gamma_{0}}{4}\left(1+\nu \gamma_{0} \lambda_{1} \Delta t\right)^{-k}\left\|\Phi^{k-1}\right\|^{2} \\
+ & \frac{4}{\nu \gamma_{0} \lambda_{1}}\left(1+\nu \gamma_{0} \lambda_{1} \Delta t\right)^{k}\left|\xi^{k}\right|^{2} \\
2\left|b\left(\Phi^{k-1}, u_{m}^{k-1}, \Phi^{k-1}\right)\right| & \leq \frac{v \gamma_{0}}{2}\left\|\Phi^{k-1}\right\|^{2} \\
& +\frac{2}{\nu \gamma_{0}} c_{0}^{2}\left|\Phi^{k-1}\right|^{2}\left\|u^{k-1}\right\|^{2} .
\end{aligned}
$$

Combining above inequalities and using (14), we obtain

$$
\begin{aligned}
(1+ & \left.\nu \gamma_{0} \lambda_{1} \Delta t\right)^{-(k-1)}\left|\Phi^{k-1}\right|^{2}-\left(1+\nu \gamma_{0} \lambda_{1} \Delta t\right)^{-k}\left|\Phi^{k}\right|^{2} \\
& +\frac{\nu \gamma_{0}}{4}\left(1+\nu \gamma_{0} \lambda_{1} \Delta t\right)^{-k}\left\|\Phi^{k-1}\right\|^{2} \Delta t \\
\leq & \frac{2}{\nu \gamma_{0}} c_{0}^{2}\left(1+\nu \gamma_{0} \lambda_{1} \Delta t\right)^{-k}\left\|u_{m}^{k-1}\right\|^{2}\left|\Phi^{k-1}\right|^{2} \Delta t \\
& +\frac{4}{\nu \gamma_{0} \lambda_{1}}\left(1+\nu \gamma_{0} \lambda_{1} \Delta t\right)^{k}\left|\xi^{k}\right|^{2} \Delta t
\end{aligned}
$$

Summing (158) from $r+1$ to $n$, noting that $\Phi^{n}=0$, and using Theorem 13, we arrive at

$$
\begin{aligned}
(1+ & \left.\nu \gamma_{0} \lambda_{1} \Delta t\right)^{-r}\left|\Phi^{r}\right|^{2} \\
& +\frac{\nu \gamma_{0}}{4} \Delta t \sum_{k=r}^{n}\left(1+\nu \gamma_{0} \lambda_{1} \Delta t\right)^{-(k+1)}\left\|\Phi^{k}\right\|^{2} \\
\leq & \frac{2}{\nu \gamma_{0}} c_{0}^{2} \Delta t \sum_{k=r}^{n}\left(1+\nu \gamma_{0} \lambda_{1} \Delta t\right)^{-k}\left\|u_{m}^{k}\right\|^{2}\left|\Phi^{k}\right|^{2} \\
& +\frac{4}{\nu \gamma_{0} \lambda_{1}} \Delta t \sum_{k=r}^{n}\left(1+\nu \gamma_{0} \lambda_{1} \Delta t\right)^{k}\left|\xi^{k}\right|^{2}
\end{aligned}
$$


for all $0 \leq r \leq n$. Let

$$
\begin{gathered}
a_{r}=\left(1+\nu \gamma_{0} \lambda_{1} \Delta t\right)^{-r}\left|\Phi^{r}\right|^{2}, \\
b_{r}=\frac{\nu \gamma_{0}}{4}\left(1+\nu \gamma_{0} \lambda_{1} \Delta t\right)^{-(r+1)}\left\|\Phi^{r}\right\|^{2}, \\
h_{r}=\frac{4}{\nu \gamma_{0} \lambda_{1}}\left(1+\nu \gamma_{0} \lambda_{1} \Delta t\right)^{r}\left|\xi^{k}\right|^{2}, \\
g_{r}=\frac{2}{v \gamma_{0}} c_{0}^{2}\left\|u_{m}^{k}\right\|^{2}, \quad \beta=0,
\end{gathered}
$$

in (159) to obtain

$$
a_{r}+\Delta t \sum_{k=r}^{n} b_{k} \leq \Delta t \sum_{k=r}^{n} g_{k} a_{k}+\Delta t \sum_{k=r}^{n} h_{k} \quad \forall 0 \leq r \leq n,
$$

with $\sigma_{r}=\left(1-g_{r} \Delta t\right)^{-1} \leq 2$. Applying Lemma 6 to this inequality yields

$$
\begin{aligned}
(1 & \left.+\nu \gamma_{0} \lambda_{1} \Delta t\right)^{-r}\left|\Phi^{r}\right|^{2}+\frac{\nu \gamma_{0}}{2} \Delta t \sum_{k=r}^{n}\left(1+\nu \gamma_{0} \lambda_{1} \Delta t\right)^{-(k+1)}\left\|\Phi^{k}\right\|^{2} \\
& \leq \mathscr{K} \exp \left(\frac{4}{\nu \gamma_{0}} c_{0}^{2} \Delta t \sum_{k=1}^{n}\left\|u_{m}^{k}\right\|^{2}\right) \Delta t \sum_{k=r}^{n}\left(1+\nu \gamma_{0} \lambda_{1} \Delta t\right)^{k}\left|\xi^{k}\right|^{2}
\end{aligned}
$$

Moreover, by taking $v=-2\left(1+\nu \gamma_{0} \lambda_{1} \Delta t\right)^{-k} A \Phi^{k-1} \Delta t$ in (109), we have

$$
\begin{aligned}
-2(1 & \left.+\nu \gamma_{0} \lambda_{1} \Delta t\right)^{-k}\left(A \Phi^{k-1}, \Phi^{k}\right)_{g} \\
& +2\left(1+\nu \gamma_{0} \lambda_{1} \Delta t\right)^{-k}\left\|\Phi^{k-1}\right\|^{2} \\
& +2 \nu\left(1+\nu \gamma_{0} \lambda_{1} \Delta t\right)^{-k}\left|A \Phi^{k-1}\right|^{2} \Delta t \\
& +2 \nu\left(1+\nu \gamma_{0} \lambda_{1} \Delta t\right)^{-k}\left(C A \Phi^{k-1}, \Phi^{k-1}\right)_{g} \Delta t \\
& +2\left(1+\nu \gamma_{0} \lambda_{1} \Delta t\right)^{-k} b\left(A \Phi^{k-1}, \Phi^{k-1}, u_{m}^{k-1}\right) \Delta t \\
& +2\left(1+\nu \gamma_{0} \lambda_{1} \Delta t\right)^{-k} b\left(u_{m}^{k-1}, \Phi^{k-1}, A \Phi^{k-1}\right) \Delta t \\
= & 2\left(A \Phi^{k-1}, \xi^{k}\right)_{g} \Delta t .
\end{aligned}
$$

Using Lemma 3 and Cauchy's inequality, we deduce that

$$
\begin{gathered}
2 \nu\left(1+\nu \gamma_{0} \lambda_{1} \Delta t\right)^{-k}\left|\left(C A \Phi^{k-1}, \Phi^{k-1}\right)_{g}\right| \\
\leq 2 \nu\left(1+\nu \gamma_{0} \lambda_{1} \Delta t\right)^{-k} \frac{|\nabla g|_{\infty}}{m_{0} \lambda_{1}^{1 / 2}}\left|A \Phi^{k-1}\right|^{2} \\
+c\left(1+\nu \gamma_{0} \lambda_{1} \Delta t\right)^{-k}\left\|\Phi^{k-1}\right\|^{2} .
\end{gathered}
$$

Therefore,

$$
\begin{aligned}
2(1+ & \left.\nu \gamma_{0} \lambda_{1} \Delta t\right)^{-k}\left\|\Phi^{k-1}\right\|^{2} \\
& +2 \nu \gamma_{0}\left(1+\nu \gamma_{0} \lambda_{1} \Delta t\right)^{-k}\left|A \Phi^{k-1}\right|^{2} \Delta t \\
\leq & 2\left(A \Phi^{k-1}, \xi^{k}\right)_{g} \Delta t+2\left(1+\nu \gamma_{0} \lambda_{1} \Delta t\right)^{-k}\left(A \Phi^{k-1}, \Phi^{k}\right)_{g} \\
& +2\left(1+\nu \gamma_{0} \lambda_{1} \Delta t\right)^{-k}\left|b\left(A \Phi^{k-1}, \Phi^{k-1}, u_{m}^{k-1}\right)\right| \Delta t \\
& +2\left(1+\nu \gamma_{0} \lambda_{1} \Delta t\right)^{-k}\left|b\left(u_{m}^{k-1}, \Phi^{k-1}, A \Phi^{k-1}\right)\right| \Delta t \\
& +c\left(1+\nu \gamma_{0} \lambda_{1} \Delta t\right)^{-k}\left\|\Phi^{k-1}\right\|^{2} \Delta t .
\end{aligned}
$$

Using (17)-(19) and Cauchy's inequality, we have

$$
\begin{gathered}
2\left|b\left(A \Phi^{k-1}, \Phi^{k-1}, u_{m}^{k-1}\right)\right|+2\left|b\left(u_{m}^{k-1}, \Phi^{k-1}, A \Phi^{k-1}\right)\right| \\
\leq \frac{\nu \gamma_{0}}{4}\left|A \Phi^{k-1}\right|^{2}+c\left|u_{m}^{k-1}\right|^{2}\left\|u_{m}^{k-1}\right\|^{2}\left\|\Phi^{k-1}\right\|^{2}, \\
2\left(A \Phi^{k-1}, \Phi^{k}\right)_{g}=2\left(\left(\Phi^{k-1}, \Phi^{k}\right)\right)_{g} \leq\left\|\Phi^{k-1}\right\|^{2}+\left\|\Phi^{k}\right\|^{2}, \\
2\left(A \Phi^{k-1}, \xi^{k}\right)_{g} \leq \frac{v \gamma_{0}}{4}\left(1+\nu \gamma_{0} \lambda_{1} \Delta t\right)^{-k}\left|A \Phi^{k-1}\right|^{2} \\
+\frac{4}{\nu \gamma_{0}}\left(1+\nu \gamma_{0} \lambda_{1} \Delta t\right)^{k}\left|\xi^{k}\right|^{2} .
\end{gathered}
$$

Combining above inequality, noting that $\lambda_{1}\|v\|^{2} \leq|A v|^{2}$, we obtain

$$
\begin{aligned}
(1+ & \left.\nu \gamma_{0} \lambda_{1} \Delta t\right)^{-(k-1)}\left\|\Phi^{k-1}\right\|^{2}-\left(1+\nu \gamma_{0} \lambda_{1} \Delta t\right)^{-k}\left\|\Phi^{k}\right\|^{2} \\
& +\frac{\nu \gamma_{0}}{2}\left(1+\nu \gamma_{0} \lambda_{1} \Delta t\right)^{-k}\left|A \Phi^{k-1}\right|^{2} \Delta t \\
\leq & c\left(1+\nu \gamma_{0} \lambda_{1} \Delta t\right)^{-k}\left(\left|u_{m}^{k-1}\right|^{2}\left\|u_{m}^{k-1}\right\|^{2}+1\right)\left\|\Phi^{k-1}\right\|^{2} \Delta t \\
& +c\left(1+\nu \gamma_{0} \lambda_{1} \Delta t\right)^{k}\left|\xi^{k}\right|^{2} \Delta t
\end{aligned}
$$

for all $0 \leq k \leq n$. Summing (167) from $r+1$ to $n$, we obtain

$$
\begin{gathered}
\left(1+\nu \gamma_{0} \lambda_{1} \Delta t\right)^{-r}\left\|\Phi^{r}\right\|^{2}+\frac{\nu \gamma_{0}}{2} \Delta t \sum_{k=r}^{n}\left(1+\nu \gamma_{0} \lambda_{1} \Delta t\right)^{-(k+1)}\left|A \Phi^{k}\right|^{2} \\
\leq c \sup _{k \geq 0}\left\{\left(\left|u_{m}^{k}\right|^{2}\left\|u_{m}^{k}\right\|^{2}+1\right)\right\} \Delta t \sum_{k=r}^{n}\left(1+\nu \gamma_{0} \lambda_{1} \Delta t\right)^{-(k+1)}\left\|\Phi^{k}\right\|^{2} \\
+c \Delta t \sum_{k=r+1}^{n}\left(1+\nu \gamma_{0} \lambda_{1} \Delta t\right)^{k}\left|\xi^{k}\right|^{2} \quad \forall 0 \leq r \leq n .
\end{gathered}
$$

Combining (168) with (162) and using Theorem 13, we complete the proof. 
5.2. Error Analysis. In this subsection, we will establish the $H^{1}$ - and $L^{2}$-error estimates uniform in time for the fully discrete spectral Galerkin method with the explicit time discretization for the nonlinear term. To do this, by integrating (35) from $t_{n}$ to $t_{n+1}$, we obtain

$$
\begin{gathered}
d_{t} u_{m}\left(t_{n+1}\right)+\frac{v}{\Delta t} \int_{t_{n}}^{t_{n+1}} A u_{m}(t) d t+\frac{v}{\Delta t} \int_{t_{n}}^{t_{n+1}} C u_{m}(t) d t \\
+\frac{1}{\Delta t} \int_{t_{n}}^{t_{n+1}} P_{m} B\left(u_{m}(t), u_{m}(t)\right) d t=f^{n+1}
\end{gathered}
$$

Subtracting (106) from (153) and setting $e^{n}=u_{m}\left(t_{n}\right)-u_{m}^{n}$, we have

$$
\begin{gathered}
d_{t} e^{n+1}+\nu A e^{n+1}+\nu C e^{n+1}+P_{m} B\left(e^{n}, u_{m}^{n}\right) \\
+P_{m} B\left(u_{m}\left(t_{n}\right), e^{n}\right)=P_{m} E_{n+1},
\end{gathered}
$$

with $e^{0}=0$ and

$$
\begin{aligned}
E_{n+1}= & \frac{v}{\Delta t} \int_{t_{n}}^{t_{n+1}} A\left(u_{m}\left(t_{n+1}\right)-u_{m}(t)\right) d t \\
& +\frac{v}{\Delta t} \int_{t_{n}}^{t_{n+1}} C\left(u_{m}\left(t_{n+1}\right)-u_{m}(t)\right) d t \\
& +\frac{1}{\Delta t} \int_{t_{n}}^{t_{n+1}}\left(B\left(u_{m}\left(t_{n}\right), u_{m}\left(t_{n}\right)\right)\right. \\
= & \frac{v}{\Delta t} \int_{t_{n}}^{t_{n+1}}\left(t-t_{n}\right) A u_{m t} d t+\frac{v}{\Delta t} \int_{t_{n}}^{t_{n+1}}\left(t-t_{n}\right) C u_{m t} d t \\
& -\frac{1}{\Delta t} \int_{t_{n}}^{t_{n+1}}\left(t_{n+1}-t\right)\left(B\left(u_{m t}, u_{m}\right)+B\left(u_{m}, u_{m t}\right)\right) d t
\end{aligned}
$$

To derive a bound on $e^{n}$, we need to provide the following estimates of $E_{n}$.

Lemma 15. Under the assumptions of Theorem 13, the error $E_{n}$ satisfies the following bounds:

$$
\begin{gathered}
\Delta t \sum_{k=1}^{n}\left(1+\nu \gamma_{0} \lambda_{1}\right)^{k-1}\left|A^{-1} P_{m} E_{k}\right|^{2} \\
\leq \mathscr{K}\left(1+\nu \gamma_{0} \lambda_{1}\right)^{n} \Delta t^{2} \quad \forall n \geq 1, \\
\Delta t \sum_{k=1}^{n}\left(1+v \gamma_{0} \lambda_{1}\right)^{k-1}\left|A^{-1 / 2} P_{m} E_{k}\right|^{2} \\
\leq \mathscr{K}\left(1+v \gamma_{0} \lambda_{1}\right)^{n} \Delta t \quad \forall n \geq 1, \\
\Delta t \sum_{k=1}^{n}\left(1+v \gamma_{0} \lambda_{1}\right)^{k-1} \tau\left(t_{k}\right)\left|A^{-1 / 2} P_{m} E_{k}\right|^{2} \\
\leq \mathscr{K}\left(1+v \gamma_{0} \lambda_{1}\right)^{n} \Delta t^{2} \quad \forall n \geq 1 .
\end{gathered}
$$

Proof. In view of (17)-(19), Lemma 3, and Theorem 8, we deduce from (171) that

$$
\begin{aligned}
& \left|A^{-1} P_{m} E_{n}\right| \\
& =\sup _{v \in H_{m}} \frac{\left|\left(E_{n}, v\right)\right|}{|A v|} \\
& \leq \Delta t^{-1 / 2}\left(\int _ { t _ { n - 1 } } ^ { t _ { n } } \left(c_{1}\left(\left(t-t_{n-1}\right)^{2}+\frac{|\nabla g|_{\infty}}{m_{0}}\left(t-t_{n-1}\right)^{2}\right)\left|u_{m t}\right|^{2}\right.\right. \\
& \left.\left.+c_{2}\left(t_{n}-t\right)^{2}\left\|u_{m}\right\|^{2}\left|u_{m t}\right|^{2}\right) d t\right)^{1 / 2} \\
& \leq c \Delta t^{-1 / 2}\left(\int_{t_{n-1}}^{t_{n}}\left(\left(t-t_{n-1}\right)^{2}+\left(t_{n}-t\right)^{2}\left\|u_{m}\right\|^{2}\right)\left|u_{m t}\right|^{2} d t\right)^{1 / 2}, \\
& \left|A^{-1 / 2} P_{m} E_{n}\right|=\sup _{v \in H_{m}} \frac{\left|\left(E_{n}, v\right)\right|}{\|v\|} \\
& \leq c \Delta t^{-1 / 2} \\
& \times\left(\int_{t_{n-1}}^{t_{n}}\left(\left(t-t_{n-1}\right)^{2}+\left(t_{n}-t\right)^{2}\left\|u_{m}\right\|^{2}\right)\right. \\
& \left.\times\left\|u_{m t}\right\|^{2} d t\right)^{1 / 2} .
\end{aligned}
$$

By Theorem 8, (175), and the fact that $\left(1+v \gamma_{0} \lambda_{1}\right)^{k-1} \leq$ $e^{\nu \gamma_{0} \lambda_{1} t_{k-1}}$, we have

$$
\begin{aligned}
& \left(1+\nu \gamma_{0} \lambda_{1} \Delta t\right)^{k-1}\left|A^{-1} P_{m} E_{k}\right|^{2} \Delta t \\
& \leq \mathscr{K} \Delta t^{3}\left(1+\nu \gamma_{0} \lambda_{1} \Delta t\right)^{k-1} \quad \forall k \geq n_{0}+1, \\
& \left(1+\nu \gamma_{0} \lambda_{1} \Delta t\right)^{k-1}\left|A^{-1 / 2} P_{m} E_{k}\right|^{2} \Delta t \\
& \leq \mathscr{K} \Delta t^{3}\left(1+\nu \gamma_{0} \lambda_{1} \Delta t\right)^{k-1} \quad \forall k \geq n_{0}+1, \\
& \left(1+\nu \gamma_{0} \lambda_{1} \Delta t\right)^{k-1}\left|A^{-1} P_{m} E_{k}\right|^{2} \Delta t \\
& \leq \mathscr{K} \Delta t^{2} \int_{t_{k-1}}^{t_{k}} e^{\nu \gamma_{0} \lambda_{1} t}\left|u_{m t}\right|^{2} d t \quad \forall 1 \leq k \leq n_{0}, \\
& \left(1+\nu \gamma_{0} \lambda_{1} \Delta t\right)^{k-1}\left|A^{-1 / 2} P_{m} E_{k}\right|^{2} \Delta t \\
& \leq \mathscr{K} \Delta t^{2} \int_{t_{k-1}}^{t_{k}} e^{\nu \gamma_{0} \lambda_{1} t}\left\|u_{m t}\right\|^{2} d t \quad \forall 1 \leq k \leq n_{0} .
\end{aligned}
$$

For $n \geq n_{0}+1$, summing (176) from $n_{0}+1$ to $n$, we have

$$
\begin{gathered}
\Delta t \sum_{k=n_{0}+1}^{n}\left(1+v \gamma_{0} \lambda_{1} \Delta t\right)^{k-1}\left(\left|A^{-1} P_{m} E_{k}\right|^{2}+\left|A^{-1 / 2} P_{m} E_{k}\right|^{2}\right) \\
\leq \mathscr{K} \Delta t^{3} \sum_{k=n_{0}+1}^{n}\left(1+\nu \gamma_{0} \lambda_{1} \Delta t\right)^{k-1}
\end{gathered}
$$




$$
\begin{aligned}
& \leq \mathscr{K} \Delta t^{3}\left(1+\nu \gamma_{0} \lambda_{1} \Delta t\right)^{n_{0}} \frac{1-\left(1+\nu \gamma_{0} \lambda_{1} \Delta t\right)^{n-n_{0}}}{-\nu \gamma_{0} \lambda_{1} \Delta t} \\
& \leq \mathscr{K} \Delta t^{2}\left(1+v \gamma_{0} \lambda_{1} \Delta t\right)^{n} .
\end{aligned}
$$

For $n \leq n_{0}$, we sum (177) from 1 to $n$ and use Theorem 8 to obtain

$$
\begin{aligned}
\Delta t \sum_{k=1}^{n} & \left(1+\nu \gamma_{0} \lambda_{1} \Delta t\right)^{k-1}\left|A^{-1} P_{m} E_{k}\right|^{2} \\
& \leq \mathscr{K} \Delta t^{2} e^{\nu \gamma_{0} \lambda_{1}} \\
& \leq \mathscr{K} \Delta t^{2}\left(1+v \gamma_{0} \lambda_{1} \Delta t\right)^{n} \quad \forall n \leq n_{0},
\end{aligned}
$$

which, together with (179), gives (172).

Finally, multiplying (178) by $\tau^{j}\left(t_{k}\right), j=0,1 ; 2 \leq k \leq n_{0}$, and noting that $\Delta t \leq \tau\left(t_{k-1}\right), \tau\left(t_{k}\right) \leq 2 \tau\left(t_{k-1}\right)$, we obtain

$$
\begin{aligned}
(1 & \left.+\nu \gamma_{0} \lambda_{1} \Delta t\right)^{k-1} \tau^{j}\left(t_{k}\right)\left|A^{-1 / 2} P_{m} E_{k}\right|^{2} \Delta t \\
& \leq \mathscr{K} \Delta t^{1-j} \int_{t_{k-1}}^{t_{k}} e^{\nu \gamma_{0} \lambda_{1} t} \tau^{j}(t)\left\|u_{m t}\right\|^{2} d t \quad \forall 2 \leq k \leq n_{0} .
\end{aligned}
$$

Observing again (170) with $n=1$ and using Theorem 8 yields

$$
\tau^{j}\left(t_{1}\right)\left|A^{-1 / 2} P_{m} E_{k}\right|^{2} \Delta t \leq \mathscr{K} \sup _{0 \leq t \leq t_{1}}\left\|u_{m}(t)-u_{m}(0)\right\|^{2} \Delta t^{1-j}
$$

For $2 \leq n \leq n_{0}$, summing (181) from 2 to $n$ and using Theorem 12, we obtain

$$
\Delta t \sum_{k=2}^{n}\left(1+\nu \gamma_{0} \lambda_{1} \Delta t\right)^{k-1} \tau^{j}\left(t_{k}\right)\left|A^{-1 / 2} P_{m} E_{k}\right|^{2} \leq \mathscr{K} \Delta t^{1-j} e^{\nu \gamma_{0} \lambda_{1}}
$$

Combining (182) and (183) with (179) yields (173) and (174).

Now, we prove the following error estimate.

Lemma 16. Under the assumptions of Theorem 13, one has

$$
\begin{aligned}
\left|e^{n}\right|^{2} & +\Delta t \sum_{k=1}^{n}\left(1+\nu \gamma_{0} \lambda_{1} \Delta t\right)^{-(n-1-k)} \\
& \times\left(\frac{\nu \gamma_{0}}{8}\left\|e^{k}\right\|^{2} \Delta t+\left|e^{k}-e^{k-1}\right|^{2}\right) \\
\leq & \mathscr{K} \exp \left(\frac{8}{\nu \gamma_{0}} c_{0}^{2} \Delta t \sum_{k=1}^{n}\left\|u_{m}^{k}\right\|^{2}\right) \Delta t \quad \forall n \geq 1 .
\end{aligned}
$$

Proof. Taking the scalar product of (170) with $2 e^{n+1} \Delta t$, using (11), Lemma 3 and noting that $\lambda_{1}|v|^{2} \leq\|v\|^{2}$, we have

$$
\begin{aligned}
& \left(1+v \gamma_{0} \lambda_{1} \Delta t\right)\left|e^{n+1}\right|^{2}-\left|e^{n}\right|^{2} \\
& +\left|e^{n+1}-e^{n}\right|^{2}+v \gamma_{0}\left\|e^{n+1}\right\|^{2} \Delta t \\
& +2\left(b\left(e^{n+1}, u_{m}^{n}, e^{n+1}\right)+b\left(e^{n}-e^{n+1}, u_{m}^{n}, e^{n+1}\right)\right. \\
& \left.\quad+b\left(u_{m}\left(t_{n}\right), e^{n}-e^{n+1}, e^{n+1}\right)\right) \Delta t \\
& \leq 2\left(E_{n+1}, e^{n+1}\right) \Delta t .
\end{aligned}
$$

Using (17)-(19), we get

$$
\begin{gathered}
2\left|b\left(e^{n+1}, u_{m}^{n}, e^{n+1}\right)\right| \leq \frac{\nu \gamma_{0}}{4}\left\|e^{n+1}\right\|^{2}+\frac{4}{\nu \gamma_{0}} c_{0}^{2}\left\|u_{m}^{n}\right\|^{2}\left|e^{n+1}\right|^{2}, \\
2\left|b\left(e^{n}-e^{n+1}, u_{m}^{n}, e^{n+1}\right)\right| \\
\quad+2\left|b\left(u_{m}\left(t_{n}\right), e^{n}-e^{n+1}, e^{n+1}\right)\right| \\
\leq \frac{9 v \gamma_{0}}{16}\left\|e^{n+1}\right\|^{2}+\frac{4}{9}\left(L\left(u_{m}^{n}\right)+L\left(u_{m}\left(t_{n}\right)\right)\right)\left|e^{n+1}-e^{n}\right|^{2}, \\
2\left|\left(E_{n+1}, e^{n+1}\right)\right| \leq \frac{v \gamma_{0}}{16}\left\|e^{n+1}\right\|^{2}+\frac{16}{\nu \gamma_{0}}\left|A^{-1 / 2} P_{m} E_{n+1}\right|^{2} .
\end{gathered}
$$

Hence, by combining the above inequalities with (185) and using Theorem 8, (110) and (114), we obtain

$$
\begin{aligned}
\left(1+\nu \gamma_{0} \lambda_{1} \Delta t\right)^{n+1}\left|e^{n+1}\right|^{2}-\left(1+\nu \gamma_{0} \lambda_{1} \Delta t\right)^{n}\left|e^{n}\right|^{2} \\
+\left(1+\nu \gamma_{0} \lambda_{1} \Delta t\right)^{n}\left(\frac{\nu \gamma_{0}}{8}\left\|e^{n+1}\right\|^{2} \Delta t+\frac{1}{9}\left|e^{n+1}-e^{n}\right|^{2}\right) \\
\leq \frac{4}{\nu \gamma_{0}} c_{0}^{2}\left\|u_{m}^{n}\right\|^{2}\left(1+\nu \gamma_{0} \lambda_{1} \Delta t\right)^{n}\left|e^{n+1}\right|^{2} \Delta t \\
+\frac{16}{\nu \gamma_{0}}\left(1+\nu \gamma_{0} \lambda_{1} \Delta t\right)^{n}\left|A^{-1 / 2} P_{m} E_{n+1}\right|^{2} \Delta t
\end{aligned}
$$

for all $n \geq 1$. Summing (187) from 1 to $n-1$ and using Lemma 15, we obtain

$$
\begin{aligned}
(1+ & \left.\nu \gamma_{0} \lambda_{1} \Delta t\right)^{n}\left|e^{n}\right|^{2} \\
& +\sum_{k=1}^{n}\left(1+\nu \gamma_{0} \lambda_{1} \Delta t\right)^{k-1}\left(\frac{\nu \gamma_{0}}{8}\left\|e^{k}\right\|^{2} \Delta t+\frac{1}{9}\left|e^{k}-e^{k-1}\right|^{2}\right) \\
\leq & \frac{4}{\nu \gamma_{0}} c_{0}^{2} \Delta t \sum_{k=1}^{n}\left(1+\nu \gamma_{0} \lambda_{1} \Delta t\right)^{k-1}\left\|u_{m}^{k-1}\right\|^{2}\left|e^{k}\right|^{2} \\
& +\frac{16}{\nu \gamma_{0}} \Delta t \sum_{k=1}^{n}\left(1+\nu \gamma_{0} \lambda_{1} \Delta t\right)^{k-1}\left|A^{-1 / 2} P_{m} E_{k}\right|^{2}
\end{aligned}
$$


We set

$$
\begin{gathered}
a_{k}=\left(1+\nu \gamma_{0} \lambda_{1} \Delta t\right)^{k}\left|e^{k}\right|^{2}, \\
b_{k}=\left(1+\nu \gamma_{0} \lambda_{1} \Delta t\right)^{k-1}\left(\frac{\nu \gamma_{0}}{8}\left\|e^{k}\right\|^{2}+\frac{1}{9}\left|e^{k}-e^{k-1}\right|^{2} \Delta t^{-1}\right), \\
g_{k}=\frac{4}{\nu \gamma_{0}} c_{0}^{2}\left\|u_{m}^{k-1}\right\|^{2}, \\
h_{k}=\frac{16}{\nu \gamma_{0}} \Delta t\left(1+\nu \gamma_{0} \lambda_{1} \Delta t\right)^{k-1}\left|A^{-1 / 2} P_{m} E_{k}\right|^{2}, \quad \beta=0
\end{gathered}
$$

in (188) to obtain

$$
a_{n}+\Delta t \sum_{k=1}^{n} b_{k} \leq \Delta t \sum_{k=1}^{n} g_{k} a_{k}+\Delta t \sum_{k=1}^{n} h_{k}
$$

with $\sigma_{k}=\left(1-g_{k} \Delta t\right)^{-1} \leq 2$. Applying Lemma 6 to (190) and using Lemma 15, we deduce that

$$
\begin{aligned}
(1+ & \left.\nu \gamma_{0} \lambda_{1} \Delta t\right)^{n}\left|e^{n}\right|^{2} \\
& +\sum_{k=1}^{n}\left(1+\nu \gamma_{0} \lambda_{1} \Delta t\right)^{k-1}\left(\frac{v \gamma_{0}}{8}\left\|e^{k}\right\|^{2} \Delta t+\frac{1}{9}\left|e^{k}-e^{k-1}\right|^{2}\right) \\
\leq & \left(1+\nu \gamma_{0} \lambda_{1} \Delta t\right)^{n} \mathscr{K} \exp \left(\frac{8}{\nu \gamma_{0}} c_{0}^{2} \Delta t \sum_{k=0}^{n-1}\left\|u_{m}^{k}\right\|^{2}\right) \Delta t
\end{aligned}
$$

Multiplying (191) by $\left(1+\nu \gamma_{0} \lambda_{1} \Delta t\right)^{-n}$, we get (184).

Lemma 17. Under the assumptions of Theorem 13, one has

$$
\begin{aligned}
\tau\left(t_{n}\right) & \left|u_{m}\left(t_{n}\right)-u_{m}^{n}\right|^{2} \\
& \leq \mathscr{K} \exp \left(\frac{16}{\nu \gamma_{0}} c_{0}^{2} \Delta t \sum_{k=0}^{n-1}\left\|u_{m}^{k}\right\|^{2}\right) \Delta t^{2} \quad \forall n \geq 1 .
\end{aligned}
$$

Proof. Replacing $n+1$ by $k$ in (170) and taking the scalar product of (170) with $\Phi^{k-1}$, we obtain

$$
\begin{gathered}
\left(d_{t} e^{k}, \Phi^{k-1}\right)_{g}+a\left(e^{k}, \Phi^{k-1}\right)+v\left(C e^{k}, \Phi^{k-1}\right)_{g} \\
+b\left(e^{k-1}, u_{m}^{k-1}, \Phi^{k-1}\right)+b\left(u_{m}^{k-1}, e^{k-1}, \Phi^{k-1}\right) \\
+b\left(e^{k-1}, e^{k-1}, \Phi^{k-1}\right)=\left(E_{k}, \Phi^{k-1}\right)_{g}
\end{gathered}
$$

Next, setting $v=e^{k}, \xi^{k}=e^{k}, 1 \leq k \leq n$ in (109), we get

$$
\begin{aligned}
\left(e^{k}, d_{t} \Phi^{k}\right)_{g}-a\left(e^{k}, \Phi^{k-1}\right)-v\left(C e^{k}, \Phi^{k-1}\right)_{g} & -b\left(e^{k}, u_{m}^{k-1}, \Phi^{k-1}\right)-b\left(u_{m}^{k-1}, e^{k}, \Phi^{k}\right) \\
= & \left(1+\nu \gamma_{0} \lambda_{1} \Delta t\right)^{k}\left|\xi^{k}\right|^{2} .
\end{aligned}
$$

Adding (193) to (194), we arrive at

$$
\begin{aligned}
(1+ & \left.v \gamma_{0} \lambda_{1} \Delta t\right)^{k}\left|\xi^{k}\right|^{2} \\
= & \frac{1}{\Delta t}\left(\left(e^{k}, \Phi^{k}\right)_{g}-\left(e^{k-1}, \Phi^{k-1}\right)_{g}\right) \\
& +b\left(e^{k-1}-e^{k}, u_{m}^{k-1}, \Phi^{k-1}\right)+b\left(u_{m}^{k-1}, e^{k-1}-e^{k}, \Phi^{k-1}\right) \\
& +b\left(e^{k-1}, e^{k-1}, \Phi^{k-1}\right)-\left(E_{k}, \Phi^{k-1}\right)_{g}
\end{aligned}
$$

From (17) and (18), noting that $\lambda_{1}|v|^{2} \leq\|v\|^{2}$, one finds that

$$
\begin{gathered}
\left|b\left(e^{k-1}-e^{k}, u_{m}^{k-1}, \Phi^{k-1}\right)+b\left(u_{m}^{k-1}, e^{k-1}-e^{k}, \Phi^{k-1}\right)\right| \\
\leq c\left|e^{k}-e^{k-1}\right||| u_{m}^{k-1} \|\left|A \Phi^{k-1}\right|, \\
\left|b\left(e^{k-1}, e^{k-1}, \Phi^{k-1}\right)\right| \leq c\left\|e^{k-1}\right\|\left|e^{k-1}\right|\left|A \Phi^{k-1}\right|, \\
\left|\left(E_{k}, \Phi^{k-1}\right)_{g}\right| \leq\left|A^{-1} P_{m} E_{k}\right|\left|A \Phi^{k-1}\right| .
\end{gathered}
$$

Combining (195) with the above estimates yields

$$
\begin{aligned}
(1+ & \left.\nu \gamma_{0} \lambda_{1} \Delta t\right)^{k}\left|\xi^{k}\right|^{2} \Delta t \\
\leq & \left(\left(e^{k}, \Phi^{k}\right)_{g}-\left(e^{k-1}, \Phi^{k-1}\right)_{g}\right)+\left|A^{-1} P_{m} E_{k}\right|\left|A \Phi^{k-1}\right| \Delta t \\
& +c\left(\left|e^{k}-e^{k-1}\right|\left\|u_{m}^{k-1}\right\|+\left\|e^{k-1}\right\|\left|e^{k-1}\right|\right)\left|A \Phi^{k-1}\right| \Delta t
\end{aligned}
$$

with $e^{0}=\Phi^{n}=0$. Summing (197) from 1 to $n$ and using Lemma 15, we obtain

$$
\begin{aligned}
& \Delta t \sum_{k=1}^{n}\left(1+\nu \gamma_{0} \lambda_{1} \Delta t\right)^{k}\left|\xi^{k}\right|^{2} \\
& \leq \mathscr{K} \Delta t \sum_{k=1}^{n}\left(1+v \gamma_{0} \lambda_{1} \Delta t\right)^{k} \\
& \quad \times\left(\left|e^{k}-e^{k-1}\right|^{2}\left\|u_{m}^{k-1}\right\|^{2}+\left\|e^{k-1}\right\|^{2}\left|e^{k-1}\right|^{2}\right) \\
& \quad+\Delta t \sum_{k=1}^{n}\left(1+v \gamma_{0} \lambda_{1} \Delta t\right)^{k}\left|A^{-1} P_{m} E_{k}\right|^{2} .
\end{aligned}
$$

Applying Lemmas 15 and 16 and Theorem 8 in (198), we get

$$
\begin{aligned}
& \left(1+\nu \gamma_{0} \lambda_{1} \Delta t\right)^{-n} \Delta t \sum_{k=1}^{n}\left(1+\nu \gamma_{0} \lambda_{1} \Delta t\right)^{k}\left|\xi^{k}\right|^{2} \\
& \quad \leq \mathscr{K} \exp \left(\frac{16}{\nu \gamma_{0}} c_{0}^{2} \Delta t \sum_{n=0}^{n-1}\left\|u_{m}^{k}\right\|^{2}\right) \Delta t^{2} \quad \forall n \geq 1 .
\end{aligned}
$$

Now, multiplying (187) by $\tau\left(t_{n+1}\right)$ and noting that

$$
\tau\left(t_{n+1}\right) \leq \Delta t+\tau\left(t_{n}\right), \quad \Delta t \leq \tau\left(t_{n}\right) \quad \forall 1 \leq n \leq N, e^{0}=0,
$$


we arrive at

$$
\begin{aligned}
(1+ & \left.\nu \gamma_{0} \lambda_{1} \Delta t\right)^{n+1} \tau\left(t_{n+1}\right)\left|e^{n+1}\right|^{2} \\
& -\left(1+v \gamma_{0} \lambda_{1} \Delta t\right)^{n} \tau\left(t_{n}\right)\left|e^{n}\right|^{2} \\
\leq & \mathscr{K}\left(1+v \gamma_{0} \lambda_{1} \Delta t\right)^{n}\left|e^{n}\right|^{2} \Delta t \\
& +k\left(1+v \gamma_{0} \lambda_{1} \Delta t\right)^{n} \tau\left(t_{n+1}\right)\left|A^{-1 / 2} P_{m} E_{n+1}\right|^{2} \Delta t .
\end{aligned}
$$

Summing (201) from 0 to $n-1$ and using (199) and (174), we obtain, after a final multiplication by $\left(1+v \gamma_{0} \lambda_{1} \Delta t\right)^{-n}$, the estimate (192).

Finally, combining Theorems 12, 8, and 13 with Lemma 17, we obtain the error estimate of the numerical solution $\left\{u_{m}^{n}\right\}$.

Theorem 18. Under the assumptions of Theorem 13, the following error estimates holds:

$$
\begin{array}{r}
\left|u\left(t_{n}\right)-u_{m}^{n}\right|^{2} \leq \mathscr{K} \tau^{-1}\left(t_{n}\right) e^{\left(16 / v^{3} \gamma_{0}^{3} \lambda_{1}\right) c_{0}^{2} C_{f}^{2} t_{n}}\left(\lambda_{m+1}^{-2}+\Delta t^{2}\right) \\
\forall n \geq 1 .
\end{array}
$$

\section{Acknowledgment}

This work is supported by a grant from the Le Quy Don Technical University.

\section{References}

[1] J. Roh, "Derivation of the $g$-Navier-Stokes equations," Journal of the Chungcheong Mathematical Society, vol. 19, pp. 213-218, 2006.

[2] C. T. Anh and D. T. Quyet, "Long-time behavior for 2D non-autonomous $g$-Navier-Stokes equations," Annales Polonici Mathematici, vol. 103, no. 3, pp. 277-302, 2012.

[3] C. T. Anh, D. T. Quyet, and D. T. Tinh, "Existence and finite time approximation of strong solutions to $2 \mathrm{D} g$-Navier-Stokes equations," Acta Mathematica Vietnamica, vol. 38, 2013.

[4] H.-O. Bae and J. Roh, "Existence of solutions of the $g$-NavierStokes equations," Taiwanese Journal of Mathematics, vol. 8, no. 1, pp. 85-102, 2004.

[5] J. Jiang and Y. Hou, "The global attractor of $g$-Navier-Stokes equations with linear dampness on $R^{2}$," Applied Mathematics and Computation, vol. 215, no. 3, pp. 1068-1076, 2009.

[6] M. Kwak, H. Kwean, and J. Roh, "The dimension of attractor of the 2D g-Navier-Stokes equations," Journal of Mathematical Analysis and Applications, vol. 315, no. 2, pp. 436-461, 2006.

[7] H. Kwean and J. Roh, "The global attractor of the 2D $g$-NavierStokes equations on some unbounded domains," Communications of the Korean Mathematical Society, vol. 20, no. 4, pp. 731749, 2005.

[8] J. Roh, "Dynamics of the $g$-Navier-Stokes equations," Journal of Differential Equations, vol. 211, no. 2, pp. 452-484, 2005.

[9] J. Roh, "Convergence of the $g$-Navier-Stokes equations," Taiwanese Journal of Mathematics, vol. 13, no. 1, pp. 189-210, 2009.
[10] Y. He and Y. Hou, "Galerkin and subspace decomposition methods in space and time for the Navier-Stokes equations," Nonlinear Analysis: Theory, Methods \& Applications, vol. 74, no. 10, pp. 3218-3231, 2011.

[11] Y. He, "Stability and error analysis for a spectral Galerkin method for the Navier-Stokes equations with $H^{2}$ or $H^{1}$ initial data," Numerical Methods for Partial Differential Equations, vol. 21, no. 5, pp. 875-904, 2005.

[12] J. Shen, "Long time stability and convergence for fully discrete nonlinear Galerkin methods," Applicable Analysis, vol. 38, no. 4, pp. 201-229, 1990.

[13] J. B. Burie and M. Marion, "Multilevel methods in space and time for the Navier-Stokes equations," SIAM Journal on Numerical Analysis, vol. 34, no. 4, pp. 1574-1599, 1997.

[14] M. Marion and R. Temam, "Navier-Stokes equations: theory and approximation," in Handbook of Numerical Analysis, Vol. VI, P. G. Ciarlet and J. L. Lions, Eds., pp. 503-688, NorthHolland, Amsterdam, The Netherlands, 1998.

[15] R. Temam, Navier-Stokes Equations: Theory and Numerical Analysis, vol. 2 of Studies in Mathematics and Its Applications, North-Holland Publishing, Amsterdam, The Netherlands, 2nd edition, 1979.

[16] R. Temam, Navier-Stokes Equations and Nonlinear Functional Analysis, vol. 66 of CBMS-NSF Regional Conference Series in Applied Mathematics, Society for Industrial and Applied Mathematics (SIAM), Philadelphia, Pa, USA, 2nd edition, 1995.

[17] J. G. Heywood and R. Rannacher, "Finite-element approximation of the nonstationary Navier-Stokes problem. IV. Error analysis for second-order time discretization," SIAM Journal on Numerical Analysis, vol. 27, no. 2, pp. 353-384, 1990. 




Advances in

Operations Research

mansans

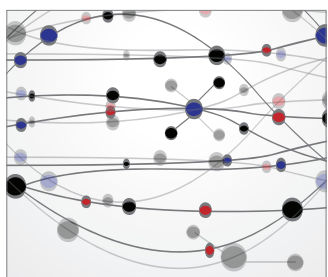

The Scientific World Journal
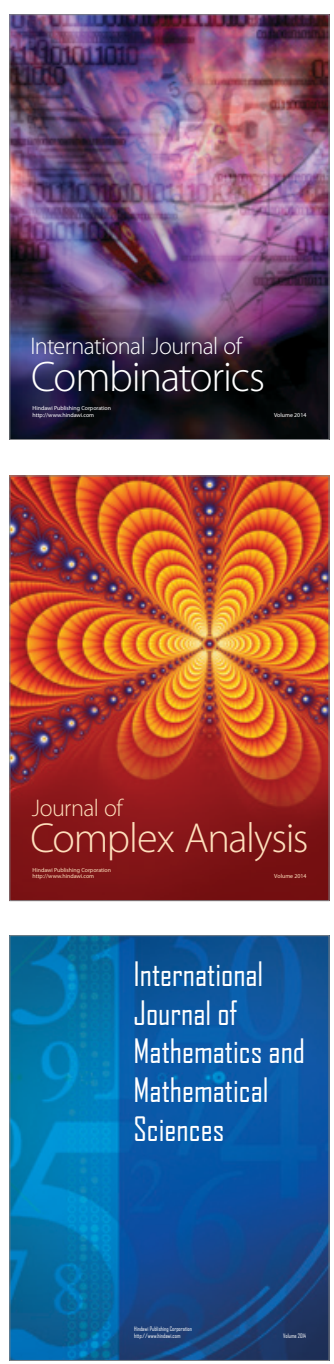
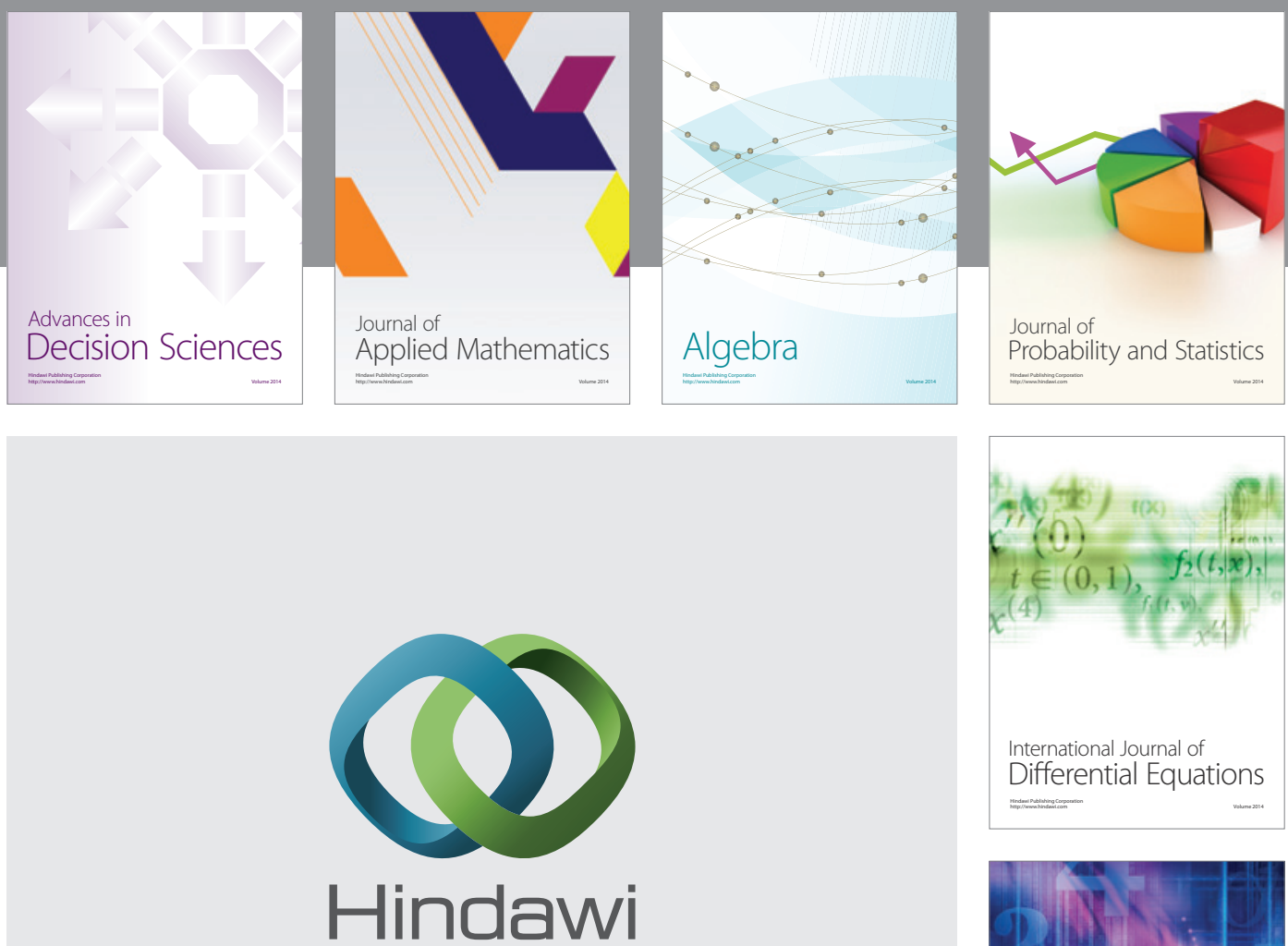

Submit your manuscripts at http://www.hindawi.com
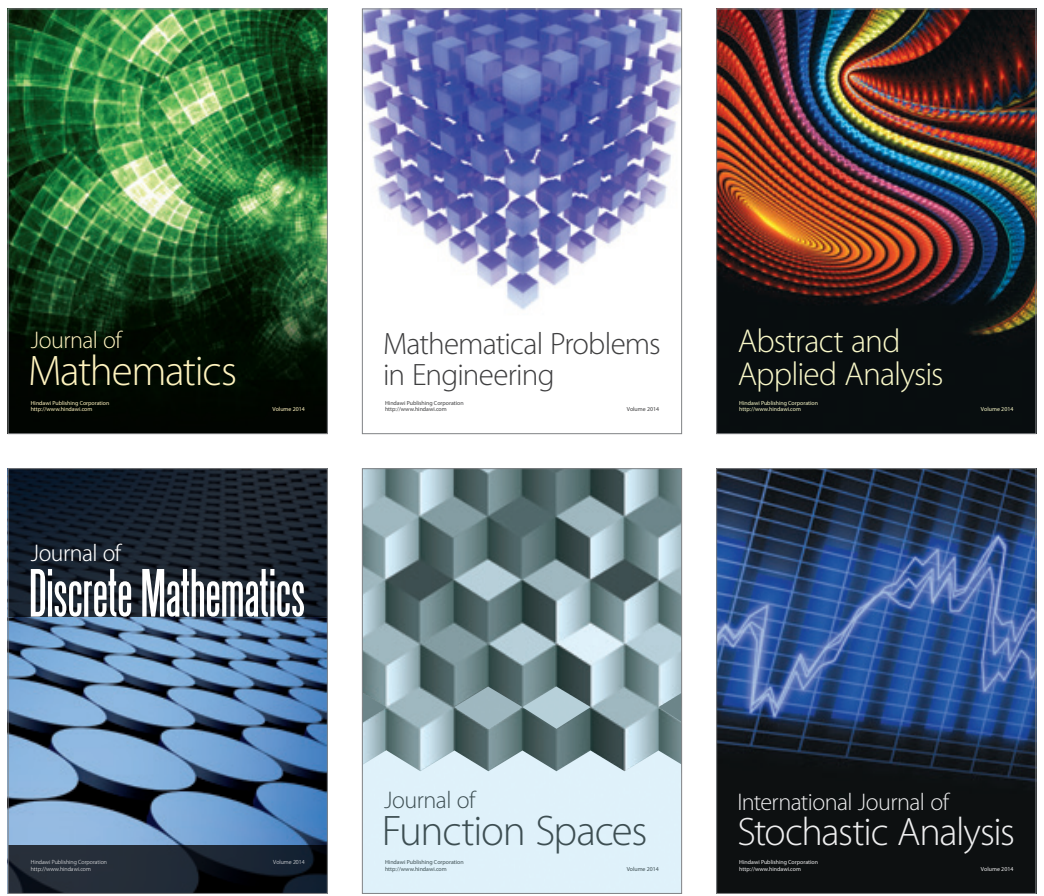

Journal of

Function Spaces

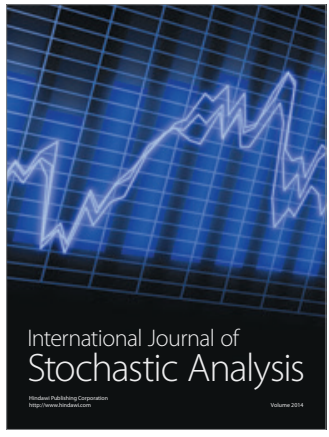

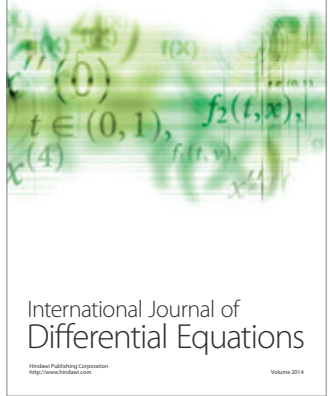
